\title{
Bivalent chromatin as a therapeutic target in cancer: An in silico predictive approach for combining epigenetic drugs
}

\section{Supplementary information}

Tomás Alarcón ${ }^{1,2,3^{*}}$ Josep Sardanyés $^{2}$, Antoni Guillamon ${ }^{4,2,5}$, Javier A. Menendez ${ }^{6,7}$,

1 ICREA, Pg. Lluís Companys 23, 08010 Barcelona, Spain.

2 Centre de Recerca Matemàtica, Edifici C, Campus de Bellaterra, 08193 Cerdanyola del Vallès (Barcelona), Spain.

3 Departament de Matemàtiques, Universitat Autònoma de Barcelona, 08193 Cerdanyola del Vallès (Barcelona), Spain.

4 Departament de Matemàtiques, Universitat Politècnica de Catalunya, Av. Gregorio Marañón 44-50, 08028 Barcelona, Spain. 5 Institut de Matemàtiques de la UPC-BarcelonaTech (IMTech), Universitat Politècnica de Catalunya, Pau Gargallo 14, 08028 Barcelona, Spain.

6 Program Against Cancer Therapeutic Resistance (ProCURE), Metabolism and Cancer Group, Catalan Institute of Oncology, 17007 Girona, Spain.

7 Girona Biomedical Research Institute, 17190 Salt, Girona, Spain.

\section{CONTENTS}

S.1 Supplementary materials and methods 1

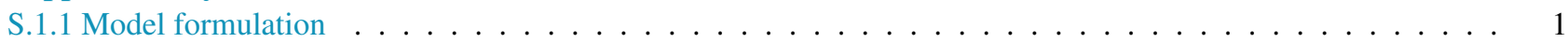

S.1.2 Asymptotic model reduction and singular perturbation analysis . . . . . . . . . . . . . . . . . 4

S.1.2.1 Asymptotic reduction of the stochastic model . . . . . . . . . . . . . . . . . 5

S.1.2.2 Asymptotic analysis of the forward equation of the reduced stochastic model for monomeric TF . . 8

S.1.3 Deterministic model: Basins of attraction and bistability . . . . . . . . . . . . . . . . . 10

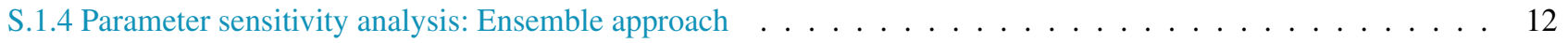

S.1.4.1 Ensemble description . . . . . . . . . . . . . . . . . . . . . . . 12

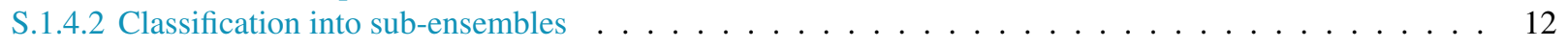

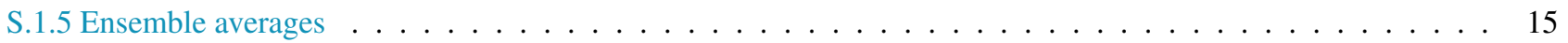

S.2 Supplementary Tables and Figures 16

\section{S.1 SUPPLEMENTARY MATERIALS AND METHODS}

\section{S.1.1 Model formulation}

3 Variables. The variables considered in our model are given by:

- $S_{A}$ : unmarked activating histone residues (H3K4).

- $S_{I}$ : unmarked repressing histone residues (H3K27).

- $E_{A}$ histone-modifying enzymes (histone methyltransferases, HMTs) that add marks (methylate) to H3K4 (MLL2).

- $E_{I}$ : histone-modifying enzymes (HMTs) that add marks (methylate) to H3K27 (EZH2).

- $E_{P_{A}}$ histone-modifying enzymes (histone demethylases, HDMs) that remove methylation marks from H3K4me3.

- $E_{P_{I}}$ : histone-modifying enzymes (HDMs) that remove methylation marks from H3K27me3.

- $C_{A}$ : complex that results from the methylation of the residue $S_{A}$ by means of the enzyme $E_{A}$.

- $C_{I}$ : complex that results from the methylation of the residue $S_{I}$ by means of the enzyme $E_{I}$.

- $P_{A}$ : marked activating substrates (trimethylated H3K4, H3K4me3).

- $P_{I}$ : marked repressing substrates (trimethylated H3K27, H3K27me3). 
Alarcón et al.

- $C_{P_{A}}$ : complex that results from the demethylation of the residue $P_{A}$ by means of the enzyme $E_{P_{A}}$.

15

16

${ }_{9}$ Dynamics. The epigenetic regulation (ER) model consists of four enzymatic reactions, which we model by means of 20 the Michaelis-Menten (MM) model of enzymatic catalysis $(1,2)$. The first reaction consists of the addition of a tri-methyl ${ }_{21}$ modification (me3) to the unmarked lysine residue H3K4 $\left(S_{A}\right)$ by means of the corresponding enzyme ( $E_{A}$ which corresponds ${ }_{22}$ to MLL). The final product of such reaction is the trimethylated residue $\mathrm{H} 3 \mathrm{~K} 4 \mathrm{me} 3$, which we denote as $P_{A}$ :

$$
S_{A}+E_{A} \underset{k_{2}\left(P_{A}, X\right)}{\stackrel{k_{1}\left(P_{A}, X\right)}{\longrightarrow}} C_{A} \stackrel{k_{3}\left(P_{A}, X\right)}{\longrightarrow} P_{A}+E_{A} .
$$

${ }_{23}$ The second reaction is the enzyme catalysed removal of me 3 from a $\mathrm{H} 3 \mathrm{~K} 4 \mathrm{me} 3$ residue. In this case the enzyme responsible for ${ }_{24}$ such reaction is referred to as $E_{P_{A}}$ and corresponds to the histone demethylase KDM:

$$
P_{A}+E_{P_{A}} \underset{k_{5}}{\stackrel{k_{4}}{\rightleftharpoons}} C_{P_{A}} \stackrel{k_{6}}{\longrightarrow} S_{A}+E_{P_{A}} .
$$

25 The third reaction consists of the addition of a tri-methyl modification (me3) to the unmarked lysine residue H3K27 $\left(S_{I}\right)$ ${ }_{26}$ mediated by the action of the associated enzyme (EZH2 which we denote as $\left.E_{I}\right)$. The final product of such reaction is the ${ }_{27}$ trimethylated residue $\mathrm{H} 3 \mathrm{~K} 27 \mathrm{me} 3$, which we refer to as $P_{I}$ :

$$
S_{I}+E_{I} \underset{k_{8}\left(P_{I}, X\right)}{\stackrel{k_{7}\left(P_{I}\right)}{\longrightarrow}} C_{I} \stackrel{k_{9}\left(P_{I}\right)}{\longrightarrow} P_{I}+E_{I} .
$$

${ }_{28}$ Finally, the last reaction is the enzyme-catalysed removal of me3 from a H3K27me3 residue. The KDM enzyme responsible 29 for catalysing such reaction is referred to as $E_{P_{I}}$ :

$$
P_{I}+E_{P_{I}} \underset{k_{11}}{\stackrel{k_{10}}{\rightleftharpoons}} C_{P_{I}} \stackrel{k_{12}}{\longrightarrow} S_{I}+E_{P_{I}} .
$$

30 Note that some of the reaction rates are not constant. They are considered to be functions of $P_{A}, P_{I}$ and the number of ${ }_{31}$ TF molecules, $X$, since the MM reactions must be supplemented with key feedback mechanisms (3-5). Such feedbacks ${ }_{32}$ are originated by the recruitment of epigenetic enzymes to unmodified sites by both epigenetic marks (6) and TFs (4), and ${ }_{33}$ have been previously modelled as regulating the reaction rates of the Michaelis-Menten reactions, $k_{i}(4,5,7,8)$. Regarding ${ }_{34}$ the coupling with the TF dynamics (see below), we consider a model whereby TFs enhances the recruitment of HME to ${ }_{35} \mathrm{H} 4 \mathrm{~K} 4 \mathrm{me} 3$ (i.e. activating substrates), $\left(k_{1}=\bar{k}_{1} X, k_{2}=\bar{k}_{2} X, k_{3}=\bar{k}_{3} X\right.$, whilst hindering the recruitment of HMEs to H3K27me3, ${ }_{36}\left(k_{7}=\bar{k}_{7}, k_{8}=\bar{k}_{8} X, k_{9}=\bar{k}_{9}\right)(4,9,10)$.

Furthermore, we consider recruitment of HMEs by modified nucleosomes. Modified nucleosomes create positive feedbacks 38 so that a particular mark favours the recruitment of enzymes that promote further addition of that mark. This positive ${ }_{39}$ feedback phenomenon is referred to as recruited histone modification. Concerning our model, we consider that methylated 40 nuclesomes recruit HMTs, which implies that the kinetic rates associated with $H M E_{A}$ and $H M E_{I}$ activity are proportional ${ }_{41}$ to the number of methylated nucleosomes $(5,7,8)$. Depending on the model of TF-induced recruitment of HME, we have ${ }_{42}\left(k_{1}=a_{1}+\hat{k}_{1} P_{A} X, k_{2}=a_{2}+\hat{k}_{2} P_{A} X, k_{3}=a_{3}+\hat{k}_{3} P_{A} X\right)$ and $\left(k_{7}=a_{7}+\hat{k}_{7} P_{I}, k_{8}=a_{8}+\hat{k}_{8} P_{I} X, k_{9}=a_{9}+\hat{k}_{9} P_{I}\right)$.

43 The gene regulation (GR) model is a simple variation of the Hill regulation model (2), and it accounts for the synthesis and ${ }_{44}$ degradation of TF molecules, $X$. We consider a basal (unregulated) rate of synthesis $R$, and a regulated synthesis mechanism. ${ }_{45}$ Such mechanism involves the binding of an $\alpha$-mer of TF molecules which binds to the free binding sites of the promoter region 46 of the gene $(F)$. The rate of synthesis is assumed to be proportional to the number of bound promoter sites, $B$. Furthermore, we ${ }_{47}$ consider degradation of the TF molecule at a constant rate $c_{2}$ :

$$
\varnothing \stackrel{R}{\longrightarrow} X, F+\alpha X \underset{c_{4}\left(P_{I}\right)}{\stackrel{c_{3}\left(P_{A}\right)}{\rightleftharpoons}} B \stackrel{c_{1}}{\longrightarrow} X \stackrel{c_{2}}{\longrightarrow} \varnothing .
$$


${ }_{48}$ The so-called Hill parameter, $\alpha$, is an integer which accounts for the cooperativity of the TF $(\alpha=1$ if the TF is monomeric, ${ }_{49} \alpha=2$ if the TF is dimeric, and so on). The coupling with the ER model is through the dependence of the rates $c_{3}$ and $c_{4}$ on $P_{A}$ so and $P_{I}: c_{3}\left(P_{A}\right)=\hat{c}_{3} P_{A}$ and $c_{4}\left(P_{I}\right)=\hat{c}_{4} P_{I}$.

${ }_{51}$ Conservation laws. The above model satisfies a number of conservation laws:

52

53 56 the system $Y:=\left(X, P_{A}, P_{I}, S_{A}, S_{I}, E_{A}, E_{P_{A}}, E_{I}, E_{P_{I}}, C_{A}, C_{P_{A}}, C_{I}, C_{P_{I}}, B\right)$. In terms of these variables, the above reactions define 16 57 transition rates that are summarised in Table A.

Table A: Transition rates associated with the stochastic dynamics of the studied gene regulatory circuit. The vector of rates $r_{i}=\left(r_{i, j}\right)=\left(\Delta_{i} X, \Delta_{i} P_{A}, \Delta_{i} P_{I}, \Delta_{i} S_{A}, \Delta_{i} S_{I}, \Delta_{i} E_{A}, \Delta_{i} E_{P_{A}}, \Delta_{i} E_{I}, \Delta_{i} E_{P_{I}}, \Delta_{i} C_{A}, \Delta_{i} C_{P_{A}}, \Delta_{i} C_{I}, \Delta_{i} C_{P_{I}}, \Delta_{i} B\right)$ defines the stoichiometry associated with reaction $i$. The entries of the vector $r_{i}$ are equal to zero unless otherwise stated in this table. For simplicity, we have dropped the hats. Since we are considering monomeric TF, $\alpha=1$.

\begin{tabular}{ll}
\hline Transition rate & Event \\
\hline$W_{1}(Y)=R+c_{1} B$ & $r_{1,1}=\Delta_{1} X=+1$ \\
$W_{2}(Y)=c_{2} X$ & $r_{2,1}=\Delta_{2} X=-1$ \\
$W_{3}(Y)=c_{3}\left(b_{0}-B\right) P_{A} X$ & $r_{3,1}=\Delta_{3} X=-1, r_{3,14}=\Delta_{3} B=+1$ \\
$W_{4}(Y)=c_{4} P_{I} B$ & $r_{4,1}=\Delta_{4} X=+1, r_{4,14}=\Delta_{4} B=-1$ \\
$W_{5}(Y)=\left(a_{1}+k_{1} P_{A} X\right) E_{A} S_{A}$ & $r_{5,4}=\Delta_{5} S_{A}=-1, r_{5,6}=\Delta_{5} E_{A}=-1, r_{5,10}=\Delta_{5} C_{A}=+1$ \\
$W_{6}(Y)=\left(a_{2}+k_{2} P_{A} X\right) C_{A}$ & $r_{6,4}=\Delta_{6} S_{A}=+1, r_{6,6}=\Delta_{6} E_{A}=+1, r_{6,10}=\Delta_{6} C_{A}=-1$ \\
$W_{7}(Y)=\left(a_{3}+k_{3} P_{A} X\right) C_{A}$ & $r_{7,2}=\Delta_{7} P_{A}=+1, r_{7,6}=\Delta_{7} E_{A}=+1, r_{7,10}=\Delta_{7} C_{A}=-1$ \\
$W_{8}(Y)=k_{4} P_{A} E_{P_{A}}$ & $r_{8,2}=\Delta_{8} P_{A}=-1, r_{8,7}=\Delta_{8} E_{P_{A}}=-1, r_{8,11}=\Delta_{8} C_{P_{A}}=+1$ \\
$W_{9}(Y)=k_{5} C_{P_{A}}$ & $r_{9,2}=\Delta_{9} P_{A}=+1, r_{9,7}=\Delta_{9} E_{P_{A}}=+1, r_{9,11}=\Delta_{9} C_{P_{A}}=-1$ \\
$W_{10}(Y)=k_{6} C_{P_{A}}$ & $r_{10,4}=\Delta_{10} S_{A}=+1, r_{10,7}=\Delta_{10} E_{P_{A}}=+1, r_{10,11}=\Delta_{10} C_{P_{A}}=-1$ \\
$W_{11}(Y)=\left(a_{7}+k_{7} P_{I}\right) E_{I} S_{I}$ & $r_{11,5}=\Delta_{11} S_{I}=-1, r_{11,8}=\Delta_{11} E_{I}=-1, r_{11,12}=\Delta_{11} C_{I}=+1$ \\
$W_{12}(Y)=\left(a_{8}+k_{8} P_{I} X\right) C_{I}$ & $r_{12,5}=\Delta_{12} S_{I}=+1, r_{12,8}=\Delta_{12} E_{I}=+1, r_{12,12}=\Delta_{12} C_{I}=-1$ \\
$W_{13}(Y)=\left(a_{9}+k_{9} P_{I}\right) C_{I}$ & $r_{13,3}=\Delta_{13} P_{I}=+1, r_{13,8}=\Delta_{13} E_{I}=+1, r_{13,12}=\Delta_{13} C_{I}=-1$ \\
$W_{14}(Y)=k_{10} P_{I} E_{P_{I}}$ & $r_{14,3}=\Delta_{14} P_{I}=-1, r_{14,9}=\Delta_{14} E_{P_{I}}=-1, r_{14,13}=\Delta_{14} C_{P_{I}}=+1$ \\
$W_{15}(Y)=k_{11} C_{P_{I}}$ & $r_{15,3}=\Delta_{15} P_{I}=+1, r_{15,9}=\Delta_{15} E_{P_{I}}=+1, r_{15,13}=\Delta_{14} C_{P_{I}}=-1$ \\
$W_{16}(Y)=k_{12} C_{P_{I}}$ & $r_{16,5}=\Delta_{16} S_{I}=+1, r_{16,9}=\Delta_{16} E_{P_{I}}=+1, r_{16,13}=\Delta_{16} C_{P_{I}}=-1$ \\
\hline
\end{tabular}

${ }_{58}$ Scaling assumptions. An essential component of our model analysis involves the characteristic scales of the system. Let ${ }_{59} E_{A}, E_{P_{A}}, E_{I}$, and $E_{P_{I}}$ be the number of $H M E_{A}, H M E_{P_{A}} H M E_{I}$, and $H M E_{P_{I}}$, respectively, and $X$ the number of TF molecules. ${ }_{60}$ We further define the following rescaled variables:

$$
S_{A}=\Omega_{S} s_{A}, S_{I}=\Omega_{S} s_{I}, P_{A}=\Omega_{S} p_{A}, P_{I}=\Omega_{S} p_{I},
$$

$$
E_{A}=\Omega_{E} E_{A}, E_{P_{A}}=\Omega_{E} E_{P_{A}}, E_{I}=\Omega_{E} E_{I}, E_{P_{I}}=\Omega_{E} E_{P_{I}},
$$$$
C_{A}=\Omega_{E} c_{A}, C_{P_{A}}=\Omega_{E} c_{p_{A}}, C_{I}=\Omega_{E} c_{I}, C_{P_{I}}=\Omega_{E} c_{p_{I}},
$$

$$
X=\Omega_{T} x, B=\Omega_{B} b, F=\Omega_{B} f .
$$

${ }_{64}$ We further make the following assumptions regarding the characteristic scales, $\Omega_{J}$ :

$$
\varepsilon_{1}=\frac{\Omega_{E}}{\Omega_{S}} \ll 1, \varepsilon_{2}=\frac{\Omega_{S}}{\Omega_{T}} \ll 1, \varepsilon_{3}=\frac{\Omega_{B}}{\Omega_{T}} \ll 1
$$

${ }_{65}$ with $\varepsilon_{3} / \varepsilon_{2}=\mathscr{O}\left(\varepsilon_{1}\right)$. The condition $\varepsilon_{1} \ll 1$ is the usual Briggs-Haldane assumption leading to the Michaelis-Menten approxi66 mation for enzyme kinetics (1). 


\section{${ }_{67}$ S.1.2 Asymptotic model reduction and singular perturbation analysis}

68 We now proceed to formulate the stochastic model of the epigenetically-regulated gene circuit. The stochastic equations 69 describing the dynamics of the model, encapsulated in the rates and stoichiometry described in Table A, are (11):

$$
Y_{j}(t)=Y_{j}(0)+\sum_{i=1}^{16} r_{i, j} \mathscr{P}\left(\int_{0}^{t} W_{i}(Y(s)) d s\right)
$$

70 where $\left.Y(t)=\left(Y_{i}(t)\right), i=1, \ldots, 14\right)$, is the state vector of the system, whose entries are the numbers of the different components 71 of the system at time $t$ (see Table A). $\mathscr{P}(\lambda)$ stands for a random number sampled from a Poisson distribution with parameter $72 \lambda$, i.e.

$$
P(\mathscr{P}(\lambda)=n)=\frac{\lambda^{n}}{n !} e^{-\lambda}
$$

${ }_{73}$ Each of the Poisson variables in Eq. (S.1) counts the number of times that reaction $i$ has occurred in the time interval $(0, t]$. ${ }_{74}$ In other words, Eq. (S.1) represents the process as a Markovian jump process, where the state of the system, $Y(t)$, changes 75 abruptly at discrete, random arrival times with exponentially distributed interarrival waiting times (11).

In order to make some analytical progress, we exploit the presence of multiple time scales in Eq. (S.1). Such a feature is 77 ubiquitous in biological systems and has been previously used to formulate efficient numerical algorithms and asymptotic 78 model reduction methods (12-24). In particular, under the scaling assumptions described in Section S.1.1, we have shown that 79 multiple time scales are present in the stochastic dynamics, and proposed asymptotic model reductions methods to simplify the 80 stochastic dynamics $(8,21,23,26)$.

81 In order to proceed with our asymptotic analysis, we consider the scaling of the state variables defined in Section S.1.1. We 82 further rescale the reaction rates $W_{i}(Y)$ according to the method described in (23), obtaining:

$$
W_{i}(Y)=k_{1} \Omega_{E} \Omega_{T} \Omega_{S}^{2} \omega_{i}(y)+\mathscr{O}\left(\Omega^{0}\right)
$$

83 where

$$
y=\left(y_{i}\right):=\left(x, p_{A}, p_{I}, s_{A}, s_{I}, e_{A}, e_{p_{A}}, e_{I}, e_{p_{I}}, c_{A}, c_{p_{A}}, c_{I}, c_{p_{I}}, b\right)
$$

84 is the rescaled state vector. Further, by defining the rescaled time variable $\tau=k_{1} \Omega_{E} \Omega_{S}^{2} t$, Eq. (S.1) reads:

$$
\begin{aligned}
& y_{1}(\tau)=y_{1}(0)+\sum_{i=1}^{16} r_{i, 1} \frac{1}{\Omega_{T}} \mathscr{P}\left(\Omega_{T} \int_{0}^{\tau} \omega_{i}(y(s)) d s\right), \\
& y_{j}(\tau)=y_{j}(0)+\sum_{i=1}^{16} r_{i, j} \frac{1}{\Omega_{S}} \mathscr{P}\left(\Omega_{S} \frac{1}{\varepsilon_{2}} \int_{0}^{\tau} \omega_{i}(y(s)) d s\right), j=2, \ldots, 5, \\
& y_{j}(\tau)=y_{j}(0)+\sum_{i=1}^{16} r_{i, j} \frac{1}{\Omega_{E}} \mathscr{P}\left(\Omega_{E} \frac{1}{\varepsilon_{1}} \frac{1}{\varepsilon_{2}} \int_{0}^{\tau} \omega_{i}(y(s)) d s\right), j=6, \ldots, 13, \\
& y_{14}(\tau)=y_{14}(0)+\sum_{i=1}^{16} r_{i, 14} \frac{1}{\Omega_{B}} \mathscr{P}\left(\Omega_{B} \frac{1}{\varepsilon_{3}} \int_{0}^{\tau} \omega_{i}(y(s)) d s\right),
\end{aligned}
$$

${ }_{85}$ where we recall that $y_{1}=x$ and $y_{14}=b$, see the state vector (S.2). From Eqs. (S.4)-(S.6) and the assumptions regarding the ${ }_{86}$ characteristic scales $\Omega_{J}$ (see Section S.1.1), it is straightforward to observe that the system exhibits separation of time scales. ${ }_{87}$ Under such scaling assumptions, we are able to distinguish three regimes:

1. A fast dynamics in which the HMEs and associated complexes reach quasi-equilibrium with the substrates, products, TF, and marks. Also, the binding sites in the promoter of the gene reach quasi-equilibrium with TFs and ER products. Such dynamics is associated with the so-called fast variables $y_{j}, j=6, \ldots, 13$ and $y_{14}=b$, see Eqs. (S.7)-(S.8) in Section S.1.2.1.

2. An intermediate dynamics in which epigenetic substrates and products reach quasi-equilibrium with $\mathrm{TF}$ and marks. This regime corresponds to the dynamics of $y_{2}=p_{A}, y_{3}=p_{I}, y_{4}=s_{A}$ and $y_{5}=s_{I}$. Using conservation laws, this dynamics is reduced to system (S.17)-(S.18) in the variables $p_{A}$ and $p_{I}$, which can be further simplified by a process of self-averaging that leads to equations (S.19)-(S.20). 


\section{2}

$$
\begin{aligned}
& 103 \text { the } \\
& 104 \text { S } \\
& 105 \text { in }
\end{aligned}
$$

\section{${ }_{106}$ S.1.2.1 Asymptotic reduction of the stochastic model}

107 We will now proceed to illustrate the details of the method of asymptotic model reduction based on multiple time scale 108 analysis of the stochastic model.

${ }_{109}$ Inner solution. We continue our analysis by performing a further rescaling of the time variable: $\mathscr{T}=\varepsilon_{1}^{-1} \varepsilon_{2}^{-1} \tau$. Upon 110 applying this rescaling, it is straightforward to see that the transition rates affecting the slow variable, $y_{1}=x$, becomes $\mathscr{O}\left(\varepsilon_{1} \varepsilon_{2}\right)$. ${ }_{111}$ Furthermore, the transition rates in the stochastic equations for $y_{2}(\mathscr{T}), y_{3}(\mathscr{T}), y_{4}(\mathscr{T})$, and $y_{5}(\mathscr{T})$ become $\mathscr{O}\left(\varepsilon_{2}\right)$. As a 112 consequence we have that $y_{1}(T)=x \simeq$ cnt., and also that $y_{2}(\mathscr{T})=p_{A} \simeq$ cnt., $y_{3}(\mathscr{T})=p_{I} \simeq$ cnt., $y_{4}(\mathscr{T})=s_{A} \simeq$ cnt., and ${ }_{113} y_{5}(\mathscr{T})=s_{I} \simeq \mathrm{cnt}$. As a result of this rescaling, the rates of the the fast variables expressed as functions of $\mathscr{T}$, become $\mathscr{O}(1)$ so 114 that:

$$
\begin{aligned}
y_{j}(\mathscr{T}) & =y_{j}(0)+\sum_{i=1}^{16} r_{i, j} \frac{1}{\Omega_{E}} \mathscr{P}\left(\Omega_{E} \int_{0}^{\mathscr{T}} \omega_{i}(y(s)) d s\right), j=6, \ldots, 13, \\
b(\mathscr{T}) & =b(0)+\sum_{i=1}^{16} r_{i, 14} \frac{1}{\Omega_{B}} \mathscr{P}\left(\Omega_{B} \varepsilon_{1} \frac{\varepsilon_{2}}{\varepsilon_{3}} \int_{0}^{\mathscr{T}} \omega_{i}(y(s)) d s\right),
\end{aligned}
$$

115 where $\varepsilon_{1} \frac{\varepsilon_{2}}{\varepsilon_{3}}=\mathscr{O}(1)$. The stochastic dynamics described by Eqs. (S.7)-(S.8) proceeds under (i.e. conditioned to) $y_{2}(\mathscr{T})=p_{A} \simeq$ 116 cnt., $y_{3}(\mathscr{T})=p_{I} \simeq$ cnt., $y_{4}(\mathscr{T})=s_{A} \simeq$ cnt., and $y_{5}(\mathscr{T})=s_{I} \simeq$ cnt., and also $y_{1}(\mathscr{T})=x \simeq$ cnt.

117 Since the fast variables relax towards their quasi-equilibrium distribution, i.e. conditioned to the instantaneous value of 118 the slower variables, we study the steady state behaviour of Eqs. (S.7)-(S.8). Such distributions have been previously studied 119 in Ref. (8). In particular, the quasi-steady state distribution (QSSD) of the fast variable $B$ is a binomial, whose probability 120 generating function (PGF) is given by:

$$
G_{B}(z)=\left(\frac{\xi_{4} p_{I}+\xi_{3} p_{A} x z}{\xi_{4} p_{I}+\xi_{3} p_{A} x}\right)^{b_{0}}
$$

${ }_{121}$ Similarly, the QSSD of each of the four HMEs is also a binomial (see (8)). The PGF for the QSSD of $H M E_{A}$ and $H M E_{P_{A}}$ 122 are given by:

$$
\begin{aligned}
& G_{1}(z)=\left(1-p_{1}\left(s_{A}, x\right)+p_{1}\left(s_{A}, x\right) z\right)^{E_{A, 0}}, \\
& G_{2}(z)=\left(1-p_{2}\left(s_{A}, x\right)+p_{2}\left(s_{A}, x\right) z\right)^{E_{P_{A}, 0}} .
\end{aligned}
$$

${ }_{123}$ The quantities $p_{1}\left(s_{A}, x\right)$ and $p_{2}\left(s_{A}, x\right)$ are defined as:

124

$$
p_{1}\left(s_{A}, x\right) \equiv \frac{\alpha_{2}+\kappa_{2}\left(p_{A}, x\right)+\alpha_{3}+\kappa_{3}\left(p_{A}, x\right)}{\left(\alpha_{1}+\kappa_{1}\left(p_{A}, x\right)\right) s_{A}+\alpha_{2}+\kappa_{2}\left(p_{A}, x\right)+\alpha_{3}+\kappa_{3}\left(p_{A}, x\right)},
$$

$$
p_{2}\left(s_{A}, x\right) \equiv \frac{\kappa_{5}+\kappa_{6}}{\kappa_{4} p_{A}+\kappa_{5}+\kappa_{6}}
$$

125 where $\kappa_{i}$ stand for the rescaled versions of their counterparts, $k_{i}$ (see Table S.1), with $\kappa_{1}\left(p_{A}, x\right)=\kappa_{1} p_{A} x, \kappa_{2}\left(p_{A}, x\right)=\kappa_{2} p_{A} x$, 126 and $\kappa_{2}\left(p_{A}, x\right)=\kappa_{3} p_{A} x$. 
Alarcón et al.

${ }_{127}$ Furthermore, since (from the conservation laws) $s_{A}+p_{A}+\varepsilon_{2}\left(c_{A}+c_{P A}\right)=\frac{S_{A, 0}}{\Omega_{S}} \equiv \rho_{A}$ and $\varepsilon_{2} \ll 1$, we have that $p_{A} \simeq \rho_{A}-s_{A}$. 128

Regarding $H M E_{I}$ and $H M E_{P_{I}}$, we have:

$$
\begin{aligned}
& G_{3}(z)=\left(\left(1-p_{3}\left(s_{I}, x\right)\right)+p_{3}\left(s_{I}, x\right) z\right)^{E_{I, 0}} \\
& G_{4}(z)=\left(\left(1-p_{4}\left(s_{I}, x\right)\right)+p_{4}\left(s_{I}, x\right) z\right)^{E_{P_{I}, 0}}
\end{aligned}
$$

129 where

$$
p_{3}\left(s_{I}, x\right) \equiv \frac{\alpha_{8}+\kappa_{8}\left(p_{I}, x\right)+\alpha_{9}+\kappa_{9}\left(p_{I}, x\right)}{\left(\alpha_{7}+\kappa_{7}\left(p_{I}, x\right)\right) s_{I}+\alpha_{8}+\kappa_{8}\left(p_{I}, x\right)+\alpha_{9}+\kappa_{9}\left(p_{I}, x\right)},
$$

${ }_{130}$ with $\kappa_{7}\left(p_{I}, x\right)=\kappa_{7} p_{I} x, \kappa_{8}\left(p_{I}, x\right)=\kappa_{8} p_{I} x$, and $\kappa_{9}\left(p_{I}, x\right)=\kappa_{3} p_{I} x$ (see Table B), and

$$
p_{4}\left(s_{I}, x\right) \equiv \frac{\kappa_{11}+\kappa_{12}}{\kappa_{10} p_{I}+\kappa_{11}+\kappa_{12}} .
$$

In this case we also have that, since (from the conservation laws) $s_{I}+p_{I}+\varepsilon_{2}\left(c_{I}+c_{P I}\right)=\frac{s_{I, 0}}{\Omega_{S}} \equiv \rho_{I}$ and $\varepsilon_{2} \ll 1, p_{I} \simeq \rho_{I}-s_{I}$.

Table B: Parameter groupings, see Materials and Methods in the main manuscript. The parameter $\rho$ is the rescaled version of $R$ : $\rho=\frac{R}{k_{1} \Omega_{E} \Omega_{T} \Omega_{S}^{2}}$.

\begin{tabular}{|c|}
\hline Methylation/demethylation of H3K4 \\
\hline$A_{1}=\frac{\alpha_{1}}{\kappa_{1}}, A_{3}=\frac{\alpha_{3}}{\kappa_{3}}, B_{2}=\frac{\alpha_{2}+\alpha_{3}}{\kappa_{1}}, K_{2}=\frac{\kappa_{2}+\kappa_{3}}{\kappa_{1}}, K_{5}=\frac{\kappa_{5}+\kappa_{6}}{\kappa_{4}}$ \\
\hline$K_{6}=\frac{\kappa_{6}}{\kappa_{5}}, \Lambda_{A}=\kappa_{3} \tilde{\rho}_{A}, \lambda_{A}=\frac{\kappa_{5} \tilde{\rho}_{I}}{\kappa_{3} \tilde{\rho}_{A}}$ \\
\hline Methylation/demethylation of H3K27 \\
\hline$A_{7}=\frac{\alpha_{7}}{\kappa_{7}}, A_{9}=\frac{\alpha_{9}}{\kappa_{9}}, B_{8}=\frac{\alpha_{8}+\alpha_{9}}{\kappa_{7}}, K_{8}=\frac{\kappa_{8}}{\kappa_{7}}, K_{9}=\frac{\kappa_{9}}{\kappa_{7}}, K_{11}=\frac{\kappa_{11}+\kappa_{12}}{\kappa_{10}}$ \\
\hline$K_{12}=\frac{\kappa_{12}}{\kappa_{11}}, \Lambda_{I}=\kappa_{9} \tilde{\rho}_{p_{A}}, \lambda_{I}=\frac{\kappa_{11} \tilde{\rho}_{p_{I}}}{\kappa_{9} \tilde{\rho}_{p_{A}}}$ \\
\hline Gene regulatory network \\
\hline$C_{1}=\frac{\beta_{0} \xi_{1}}{\rho}, C_{4}=\frac{\beta_{0} \xi_{4}}{\rho}, \lambda_{x}=\frac{\xi_{4}}{\xi_{3}}$ \\
\hline
\end{tabular}

${ }_{132}$ Intermediate solution. We continue the analyses of Eqs. (S.4)-(S.6) by rescaling the time variable so that $T=\varepsilon_{2}^{-1} \tau$. Again, ${ }_{133}$ it is straightforward to check that all the transition rates of the stochastic equations for the slow variable $y_{1}=x$ becomes $\mathscr{O}\left(\varepsilon_{2}\right)$. ${ }_{134}$ This implies that, up to leading order, $y_{1}(T)=x$ remains approximately constant. The stochastic equations for the intermediate 135 and fast variables become:

$$
\begin{aligned}
& y_{j}(T)=y_{j}(0)+\sum_{i=1}^{16} r_{i, j} \frac{1}{\Omega_{S}} \mathscr{P}\left(\Omega_{S} \int_{0}^{T} \omega_{i}(y(s)) d s\right), j=2, \ldots, 5, \\
& y_{j}(T)=y_{j}(0)+\sum_{i=1}^{16} r_{i, j} \frac{1}{\Omega_{E}} \mathscr{P}\left(\Omega_{E} \frac{1}{\varepsilon_{3}} \int_{0}^{T} \omega_{i}(y(s)) d s\right), j=6, \ldots, 13, \\
& b(T)=b(0)+\sum_{i=1}^{16} r_{i, 14} \frac{1}{\Omega_{B}} \mathscr{P}\left(\Omega_{B} \frac{\varepsilon_{2}}{\varepsilon_{3}} \int_{0}^{T} \omega_{i}(y(s)) d s\right)
\end{aligned}
$$

${ }_{136}$ where $b(T)=y_{14}(T)$. The evolution described by Eqs. (S.14)-(S.16) occurs under $y_{1}(T)=x \simeq$ cnt. Note that, by representing 137 the dynamics as a function of the variable $T$, the rates of $y_{2}=p_{A}, y_{3}=p_{I}, y_{4}=s_{A}$ and $y_{5}=s_{I}$ become $\mathscr{O}(1)$. By contrast, ${ }_{138} y_{j}(T), j=6, \ldots, 13$ and $b=y_{14}(T)$ remain as fast variables since their rates are $\mathscr{O}\left(\varepsilon_{3}^{-1}\right)$ and $\mathscr{O}\left(\frac{\varepsilon_{2}}{\varepsilon_{3}}\right)=\mathscr{O}\left(\varepsilon_{1}^{-1}\right)$, respectively. ${ }_{139}$ This implies that the latter will swiftly relax on to their QSSD, and therefore their values can be sampled from the QSSDs 
140 derived in the previous section conditioned to the instantaneous values of the slow and intermediate variables. Therefore the 141 dynamics in the intermediate regime is described by:

$$
\begin{aligned}
& p_{A}(T)=p_{A}(0)+\sum_{i=1}^{16} r_{i, 2} \frac{1}{\Omega_{S}} \mathscr{P}\left(\Omega_{S} \int_{0}^{T} \omega_{i}(y(s)) d s\right), \\
& p_{I}(T)=p_{I}(0)+\sum_{i=1}^{16} r_{i, 3} \frac{1}{\Omega_{S}} \mathscr{P}\left(\Omega_{S} \int_{0}^{T} \omega_{i}(y(s)) d s\right),
\end{aligned}
$$

142 which proceeds under constant slow variable $\left(y_{1}(T)=x \simeq \mathrm{cnt}\right.$.), and with fast variables sampled from their QSSDs:

$$
b=\Omega_{B}^{-1} \operatorname{Bin}\left(p_{b}\left(p_{A}, p_{I}, x\right), b_{0}\right),
$$

143

$$
E_{A}=\Omega_{E}^{-1} \operatorname{Bin}\left(p_{1}\left(s_{A}, x\right), E_{A, 0}\right), c_{A}=\tilde{\rho}_{A}-E_{A},
$$

144

$$
E_{P_{A}}=\Omega_{E}^{-1} \operatorname{Bin}\left(p_{2}\left(s_{A}, x\right), E_{P_{A}, 0}\right), c_{p_{A}}=\tilde{\rho}_{p_{A}}-E_{P_{A}}
$$

145

$$
E_{I}=\Omega_{E}^{-1} \operatorname{Bin}\left(p_{3}\left(s_{I}, x\right), E_{I, 0}\right), c_{I}=\tilde{\rho}_{I}-E_{I},
$$

146

$$
E_{P_{I}}=\Omega_{E}^{-1} \operatorname{Bin}\left(p_{4}\left(s_{I}, x\right), E_{P_{I}, 0}\right), c_{p_{I}}=\tilde{\rho}_{p_{I}}-E_{P_{I}},
$$

where $\tilde{\rho}_{J} \equiv \frac{E_{J, 0}}{\Omega_{E}}$, with $J \in\left\{A, P_{A}, I, P_{I}\right\}$, are defined from the conservation laws, and

$$
p_{b}\left(p_{A}, p_{I}, x\right)=\frac{\xi_{3} p_{A} x}{\xi_{4} p_{I}+\xi_{3} p_{A} x} .
$$

${ }_{147}$ We also use that $s_{A} \simeq \rho_{A}-p_{A}$ and $s_{I} \simeq \rho_{I}-p_{I}$. The fast variables thus become an additional random perturbation to the 148 (stochastic) evolution equations for $s_{A}$ and $s_{I}$.

149 An additional simplification of the dynamics within the intermediate regime is possible by taking a leaf from the book 150 of Cao et al. $(14,15)$. According to Cao et al., when separation of time scales occur, the fast reactions fire up so so many ${ }_{151}$ times (within the characteristic time scale for variation of the slower ones) that a process of self-averaging occurs. In the 152 present situation, this implies that we can approximate the fast variables by their averages over the corresponding QSSD in Eqs. ${ }_{153}$ (S.17)-(S.18). Under this assumption, Eqs. (S.17)-(S.18) can be written as:

$$
\begin{aligned}
& p_{A}(T)=p_{A}(0)+\frac{1}{\Omega_{S}} \mathscr{P}\left(\Omega_{S} \int_{0}^{T} B_{A}\left(p_{A}(s), x\right) d s\right)-\frac{1}{\Omega_{S}} \mathscr{P}\left(\Omega_{S} \int_{0}^{T} D_{A}\left(p_{A}(s), x\right) d s\right), \\
& p_{I}(T)=p_{I}(0)+\frac{1}{\Omega_{S}} \mathscr{P}\left(\Omega_{S} \int_{0}^{T} B_{I}\left(p_{I}(s), x\right) d s\right)-\frac{1}{\Omega_{S}} \mathscr{P}\left(\Omega_{S} \int_{0}^{T} D_{I}\left(p_{I}(s), x\right) d s\right),
\end{aligned}
$$

154 where (see Table B):

$$
\begin{gathered}
B_{A}\left(p_{A}, x\right)=\Lambda_{A} \frac{\left(\rho_{A}-p_{A}\right)\left(A_{1}+p_{A} x\right)\left(A_{3}+p_{A} x\right)}{\left(\rho_{A}-p_{A}\right)\left(A_{1}+p_{A} x\right)+B_{2}+K_{2} p_{A} x}, \\
D_{A}\left(p_{A}, x\right)=\Lambda_{A} \lambda_{A} \frac{K_{6} p_{A}}{p_{A}+K_{5}}, \\
B_{I}\left(p_{I}, x\right)=\Lambda_{I} \frac{\left(\rho_{I}-p_{I}\right)\left(A_{7}+p_{I} x\right)\left(A_{9}+p_{I} x\right)}{\left(\rho_{I}-p_{I}\right)\left(A_{7}+p_{I} x\right)+B_{8}+\left(K_{8} x+K_{9} x\right) p_{I}},
\end{gathered}
$$

155 and

$$
D_{I}\left(p_{I}, x\right)=\Lambda_{I} \lambda_{I} \frac{K_{12} p_{I}}{p_{I}+K_{11}} .
$$

${ }_{156}$ Eqs. (S.19)-(S.20) define two (independent) nonlinear birth-and-death processes for the evolution of $P_{A}$ and $P_{I}$. 
Alarcón et al.

${ }_{157}$ Reduced stochastic model for monomeric self-activating TF. By taking into account the results of the previous two ${ }_{158}$ sections, and by expressing the dynamics in terms of the time variable $\tau$, we can finally write the reduced stochastic system. 159 The reduced stochastic model reads:

$$
\begin{aligned}
& x(\tau)=x(0)+\frac{1}{\Omega_{T}} \mathscr{P}\left(\Omega_{T} \int_{0}^{\tau} B_{x}\left(x, p_{A}, p_{I}\right) d s\right)-\frac{1}{\Omega_{T}} \mathscr{P}\left(\Omega_{T} \int_{0}^{\tau} D_{x}\left(x, p_{A}, p_{I}\right) d s\right), \\
& p_{A}(\tau)=p_{A}(0)+\frac{1}{\Omega_{S}} \mathscr{P}\left(\Omega_{S} \frac{1}{\varepsilon_{2}} \int_{0}^{\tau} B_{A}\left(p_{A}(s), x\right) d s\right)-\frac{1}{\Omega_{S}} \mathscr{P}\left(\Omega_{S} \frac{1}{\varepsilon_{2}} \int_{0}^{\tau} D_{A}\left(p_{A}(s), x\right) d s\right), \\
& p_{I}(\tau)=p_{I}(0)+\frac{1}{\Omega_{S}} \mathscr{P}\left(\Omega_{S} \frac{1}{\varepsilon_{2}} \int_{0}^{\tau} B_{I}\left(p_{I}(s), x\right) d s\right)-\frac{1}{\Omega_{S}} \mathscr{P}\left(\Omega_{S} \frac{1}{\varepsilon_{2}} \int_{0}^{\tau} D_{I}\left(p_{I}(s), x\right) d s\right),
\end{aligned}
$$

160 where

161

$$
\begin{gathered}
B_{x}\left(x, p_{A}, p_{I}\right)=\rho\left[1+\left(C_{1}+C_{4} p_{I}\right) \frac{p_{A} x}{\lambda_{x} p_{I}+p_{A} x}\right], \\
D_{x}\left(x, p_{A}, p_{I}\right)=\rho\left[C_{2} x+C_{4} p_{I} \frac{p_{A} x}{\lambda_{x} p_{I}+p_{A} x}\right] .
\end{gathered}
$$

The mean-field model equations (see Eqs. (1)-(3) of main text) can be now derived, by considering the limit $\Omega_{T} \rightarrow \infty$ and $\Omega_{S} \rightarrow \infty$. Defining

$$
q_{J}=\lim _{\Omega_{S} \rightarrow+\infty} p_{J}, \quad J \in\{A, I\},
$$

162 and imposing that the ratio $\Omega_{S} / \Omega_{T}=\varepsilon_{2}$ remains constant, we can use the law of large numbers for Poisson random variables. ${ }_{163}$ Notice that, for simplicity, we keep the notation $x$ for the TF variable. Informally stated such law reads $\frac{1}{\Omega} \mathscr{P}(\Omega \varphi) \rightarrow \varphi$ when ${ }_{164} \Omega \rightarrow \infty$ (see reference (25) for a rigorous statement of this result). By applying this limit to the system (S.21)-(S.23) we obtain

$$
\begin{aligned}
& x(\tau)=x(0)+\int_{0}^{\tau} B_{x}\left(x, q_{A}, q_{I}\right) d s-\int_{0}^{\tau} D_{x}\left(x, q_{A}, q_{I}\right) d s, \\
& q_{A}(\tau)=q_{A}(0)+\frac{1}{\varepsilon_{2}} \int_{0}^{\tau} B_{A}\left(q_{A}(s), x\right) d s-\frac{1}{\varepsilon_{2}} \int_{0}^{\tau} D_{A}\left(q_{A}(s), x\right) d s, \\
& q_{I}(\tau)=q_{I}(0)+\frac{1}{\varepsilon_{2}} \int_{0}^{\tau} B_{I}\left(q_{I}(s), x\right) d s-\frac{1}{\varepsilon_{2}} \int_{0}^{\tau} D_{I}\left(q_{I}(s), x\right) d s .
\end{aligned}
$$

${ }_{165}$ Finally, by taking the derivative with respect to $\tau$, the mean-field deterministic model Eqs. (1)-(3) in the main text are obtained.

\section{${ }_{166}$ S.1.2.2 Asymptotic analysis of the forward equation of the reduced stochastic model for monomeric TF}

167

The forward (Master) equation is the equation satified by the probability density function $\Phi\left(x, p_{A}, p_{I}, \tau\right)$, i.e. the probability 168 density of the system (S.21)-(S.23) to be in state $\left(x, p_{A}, p_{I}\right)$ at time $\tau$, is given by (27):

$$
\frac{\partial \Phi}{\partial \tau}=\mathscr{H}_{x}\left(x, \partial_{x}\right) \Phi+\frac{1}{\varepsilon_{2}}\left(\mathscr{H}_{A}\left(p_{A}, \partial_{p_{A}}\right)+\mathscr{H}_{I}\left(p_{I}, \partial_{p_{I}}\right)\right) \Phi
$$

${ }_{169}$ where the operators $\mathscr{H}_{x}, \mathscr{H}_{A}$, and $\mathscr{H}_{I}$ are given by:

170

$$
\mathscr{H}_{x} \Phi=\Omega_{T}\left(\left(e^{\partial_{x}}-1\right) B_{x}\left(x, p_{A}, p_{I}\right) \Phi\left(x, p_{A}, p_{I}, \tau\right)+\left(e^{-\partial_{x}}-1\right) D_{x}\left(x, p_{A}, p_{I}\right) \Phi\left(x, p_{A}, p_{I}, \tau\right)\right),
$$

171

$$
\mathscr{H}_{A} \Phi=\Omega_{S}\left(\left(e^{\partial_{p_{A}}}-1\right) B_{A}\left(x, p_{A}, p_{I}\right) \Phi\left(x, p_{A}, p_{I}, \tau\right)+\left(e^{-\partial_{p_{A}}}-1\right) D_{A}\left(x, p_{A}, p_{I}\right) \Phi\left(x, p_{A}, p_{I}, \tau\right)\right),
$$

$$
\mathscr{H}_{I} \Phi=\Omega_{S}\left(\left(e^{\partial_{p_{I}}}-1\right) B_{I}\left(x, p_{A}, p_{I}\right) \Phi\left(x, p_{A}, p_{I}, \tau\right)+\left(e^{-\partial_{p_{I}}}-1\right) D_{I}\left(x, p_{A}, p_{I}\right) \Phi\left(x, p_{A}, p_{I}, \tau\right)\right)
$$

and the operator $e^{r \partial x}$ is defined as:

$$
e^{r \partial x}=\sum_{n=0}^{\infty} \frac{r^{n}}{n !} \frac{\partial^{n}}{\partial x^{n}}
$$

172 so that $e^{r \partial x} f(x)=f(x+r)$, provided $f$ is $C^{\infty}$. 
173 In order to proceed forward we propose that the PDF $\Phi\left(x, p_{A}, p_{I}, \tau\right)$ can be expanded in powers of $\varepsilon_{2}: \Phi=\Phi_{0}+\varepsilon_{2} \Phi_{1}+$ ${ }_{174} \mathscr{O}\left(\varepsilon_{2}^{2}\right)(28,29)$. By introducing such an expansion in Eq. (S.27), it is straightforward to find that $\Phi_{0}$ and $\Phi_{1}$ satisfy:

$$
\begin{aligned}
& \left(\mathscr{H}_{A}\left(p_{A}, \partial_{p_{A}}\right)+\mathscr{H}_{I}\left(p_{I}, \partial_{p_{I}}\right)\right) \Phi_{0}=0 \\
& \left(\mathscr{H}_{A}\left(p_{A}, \partial_{p_{A}}\right)+\mathscr{H}_{I}\left(p_{I}, \partial_{p_{I}}\right)\right) \Phi_{1}=\partial_{\tau} \Phi_{0}-\mathscr{H}_{x}\left(x, \partial_{x}\right) \Phi_{0} .
\end{aligned}
$$

175 We now proceed to solve Eqs. (S.28) and (S.29) order by order. Regarding $\Phi_{0}\left(x, p_{A}, p_{I}, \tau\right)$, by inspection of Eq. (S.28), we 176 assume $\Phi_{0}\left(x, p_{A}, p_{I}, \tau\right)$ to have the form $\Phi_{0}\left(x, p_{A}, p_{I}, \tau\right)=\phi_{x}(x, \tau) \phi_{A}\left(p_{A} \mid x\right) \phi_{I}\left(p_{I} \mid x\right)$. In what follows, to simplify the notation, 177 we will omit the conditioned to $x$ when we refer to $\phi_{A}$ and $\phi_{I}$. Then Eq. (S.28) becomes:

$$
\phi_{x}(x, \tau)\left(\phi_{I}\left(p_{I}\right) \mathscr{H}_{A}\left(p_{A}, \partial_{p_{A}}\right) \phi_{A}\left(p_{A}\right)+\phi_{A}\left(p_{A}\right) \mathscr{H}_{I}\left(p_{I}, \partial_{p_{I}}\right) \phi_{I}\left(p_{I}\right)\right)=0
$$

178 which implies that $\phi_{A}$ and $\phi_{I}$ satisfy:

$$
\begin{aligned}
& \mathscr{H}_{A}\left(p_{A}, \partial_{p_{A}}\right) \phi_{A}\left(p_{A}\right)=0, \\
& \mathscr{H}_{I}\left(p_{A}, \partial_{p_{I}}\right) \phi_{I}\left(p_{I}\right)=0 .
\end{aligned}
$$

179 Explicit solutions to these equations are provided by the Wentzel-Kramers-Brillouin (WKB) approximation, which holds 180 when $\Omega_{S}>>1$. Such approximations for non-linear birth-and-death processes have been thoroughly studied in the literature 181 (30, 31). To the lowest order, $\phi_{A}$ and $\phi_{I}$ are given by:

$$
\phi_{J}=\frac{\mathscr{N}_{J}}{\sqrt{B_{J}\left(p_{J}, x\right) D_{J}\left(p_{J}, x\right)}} \exp \left(-\Omega_{S} \int_{0}^{p_{J}} \log \left(\frac{D_{J}(s, x)}{B_{J}(s, x)}\right) d s\right),
$$

$182 \mathscr{N}_{A}$ and $\mathscr{N}_{I}$ being normalisation constants so that $\int_{0}^{\rho_{J}} \phi_{J} d p_{J}=1$ with $J=A, I$.

${ }_{183}$ Equation (S.28) provides no information on $\phi_{x}(x, \tau)$ and thus we need to resort to the first order Eq. (S.29), in order 184 to determine $\phi_{x}(x, \tau)$. When tackling Eq. (S.29), we find a well-known problem in singularly perturbed problems. Since $185\left(\mathscr{H}_{A}\left(p_{A}, \partial_{p_{A}}\right)+\mathscr{H}_{I}\left(p_{I}, \partial_{p_{I}}\right)\right) f=0$ has non-trivial solutions, for the corresponding inhomogeneous problem Eq. (S.29) to have 186 solution(s), its right hand side needs to satisfy additional conditions (32). Such conditions are known as solvability conditions 187 and they eventually provide the equation for determining the for of $\phi_{x}(29,32-34)$. Consider $\varphi\left(p_{A}, p_{I}\right)$ defined as:

$$
\varphi\left(p_{A}, p_{I}\right)=\left\{\begin{array}{l}
1 \text { if }\left(p_{A}, p_{I}\right) \in \mathscr{D} \\
0 \text { otherwise }
\end{array}\right.
$$

188 where $\mathscr{D}=\left[0, \rho_{A}\right] \times\left[0, \rho_{I}\right]$, and consider also its scalar product with the left hand side of Eq. (S.29):

$$
\left\langle\varphi,\left(\mathscr{H}_{A}\left(p_{A}, \partial_{p_{A}}\right)+\mathscr{H}_{I}\left(p_{I}, \partial_{p_{I}}\right)\right) \Phi_{1}\right\rangle=\left\langle\mathscr{H}^{\dagger}{ }_{A}\left(p_{A}, \partial_{p_{A}}\right) \varphi+\mathscr{H}_{I}^{\dagger}\left(p_{I}, \partial_{p_{I}}\right) \varphi, \Phi_{1}\right\rangle=0,
$$

${ }_{189}$ which follows in a straightforward way from the form of the operators $\mathscr{H}_{A}$ and $\mathscr{H}_{I}$. The scalar product is defined by: ${ }_{190}\langle\varphi, \phi\rangle=\int_{\mathscr{D}} \varphi \phi d p_{A} d p_{I}$. The solvability condition thus reads:

$$
0=\int_{\mathscr{D}} \partial_{\tau} \Phi_{0} d p_{A} d p_{I}-\int_{\mathscr{D}} \mathscr{H}_{x}\left(x, \partial_{x}\right) \Phi_{0} d p_{A} d p_{I}
$$

191 Taking into account that $\Phi_{0}\left(x, p_{A}, p_{I}, \tau\right)=\phi_{x}(x, \tau) \phi_{A}\left(p_{A}\right) \phi_{I}\left(p_{I}\right)$, this condition leads to:

$$
\partial_{\tau} \phi_{x}=\left\langle\mathscr{H}_{x}\left(x, \partial_{x}\right)\right\rangle \phi_{x}
$$

192 where

$$
\left\langle\mathscr{H}_{x}\left(x, \partial_{x}\right)\right\rangle \phi_{x}=\int_{\mathscr{D}}\left(\Omega_{T}\left(\left(e^{\partial_{x}}-1\right) B_{x}(x) \phi_{x}+\left(e^{-\partial_{x}}-1\right) D_{x}(x) \phi_{x}\right)\right) \phi_{A} \phi_{I} d p_{A} d p_{I} .
$$

${ }_{193}$ In order to provide an analytic expression of the averaged operator $\left\langle\mathscr{H}_{x}\right\rangle$, we recall the WKB approximation of $\phi_{A}$ and $\phi_{I}$, 194 Eq. (S.30). Their exponential form and the fact that we are considering the situation where $\Omega_{S}>>1$ (with $\Omega_{T}>>\Omega_{S}$ ) allows 195 us to obtain an asymptotic approximation of the integrals involved in $\left\langle B_{x}\right\rangle$ and $\left\langle D_{x}\right\rangle$ using Laplace's method (35). Under such 196 approximation, to the lowest order (36), the averaged operator $\left\langle\mathscr{H}_{x}\right\rangle$ is given by:

$$
\left\langle\mathscr{H}_{x}\left(x, \partial_{x}\right)\right\rangle \phi_{x}=\Omega_{T}\left(\left(e^{\partial_{x}}-1\right)\left\langle B_{x}\right\rangle(x) \phi_{x}+\left(e^{-\partial_{x}}-1\right)\left\langle D_{x}\right\rangle(x) \phi_{x}\right),
$$


Alarcón et al.
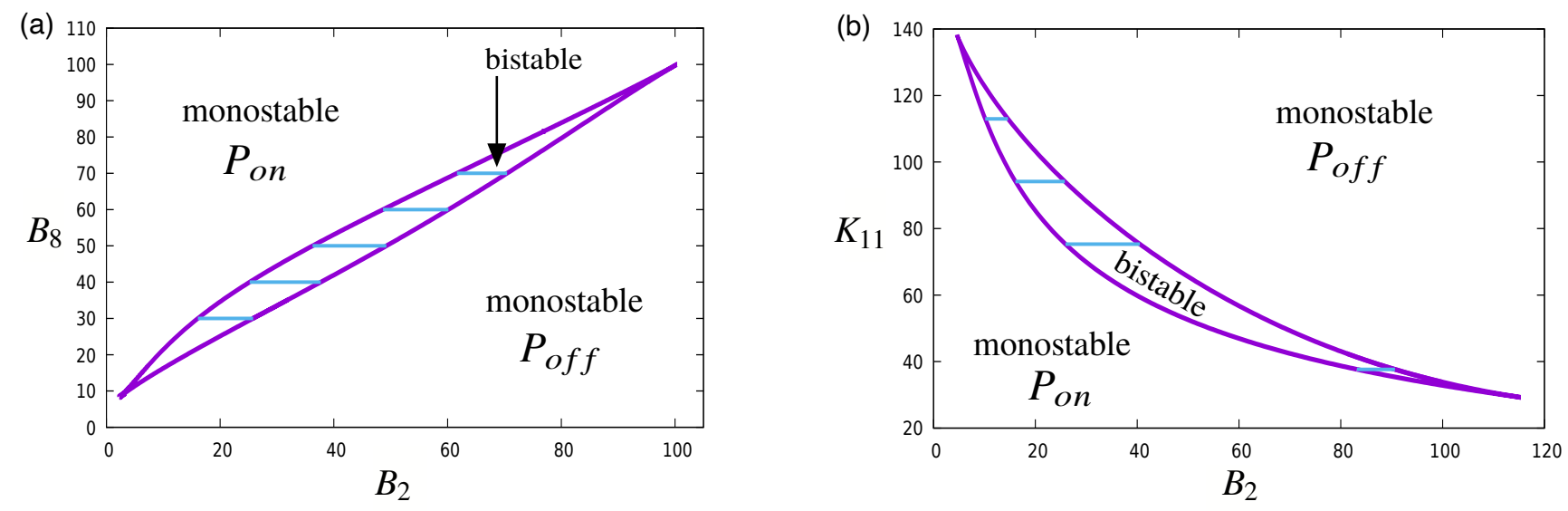

Figure A: Two-parametric bifurcation diagrams showing the curves of saddle-node bifurcations of system Eqs. (1-3) from the main text. (a) Parameter plane $\left(B_{2}, B_{8}\right)$, with $K_{11}=94.155549$. (b) Parameter plane $\left(B_{2}, K_{11}\right)$, with $B_{8}=30$. The violet solid curve represents the parameter values at which a saddle-node bifurcation occurs. The inner blue lines are specific values of $B_{8}$ for which we have computed the relative size, $P_{o f f}$, of the basin of attraction of the OFF state (see Fig. S2 below).

197 where

$$
\left\langle B_{x}\right\rangle(x)=\rho\left[1+\left(C_{1}+C_{4} q_{I}^{*}(x)\right) \frac{q_{A}^{*}(x) x}{\lambda_{x} q_{I}^{*}(x)+q_{A}^{*}(x) x}\right],
$$

198 and

$$
\left\langle D_{x}\right\rangle(x)=\rho\left[C_{2} x+C_{4} q_{I}^{*}(x) \frac{q_{A}^{*}(x) x}{\lambda_{x} q_{I}^{*}(x)+q_{A}^{*}(x) x}\right] .
$$

$199 q_{A}^{*}(x)$ and $q_{I}^{*}(x)$ are defined as:

$$
q_{A}^{*}(x)=\max _{p_{A}} \mathscr{S}_{A}\left(p_{A}, x\right) \text { and } q_{I}^{*}(x)=\max _{p_{I}} \mathscr{S}_{I}\left(p_{I}, x\right)
$$

200 with $\mathscr{S}_{J}\left(p_{J}, x\right)=\int_{0}^{p_{J}} \log \frac{D_{J}(s, x)}{B_{J}(s, x)} d s, J=A, I$. Note that $q_{A}^{*}(x)$ and $q_{I}^{*}(x)$ are the roots of the corresponding growth rates ${ }_{201} G_{J}\left(p_{J}, x\right)=B_{J}\left(p_{J}, x\right)-D_{J}\left(p_{J}, x\right)=0$, and therefore gives you the same as looking for the stationary points of the mean-field 202 equations. Recall that we are choosing parameter values (in particular, $\tilde{\rho}_{A}, \tilde{\rho}_{I}, \tilde{\rho}_{p_{A}}$, and $\tilde{\rho}_{p_{I}}$ ) so that $G_{J}\left(p_{J}, x\right)=0$ has only one 203 solution in the interval $\left[0, \rho_{J}\right]$.

204 At this point we can calculate the analytic expression of the steady state PDF $\phi_{x}(x)$ by resorting again to the WKB 205 approximation of the steady state averaged Master Equation, Eq. (S.31):

$$
\left\langle\mathscr{H}_{x}\left(x, \partial_{x}\right)\right\rangle \phi_{x}(x)=0
$$

206 which is given by (31)

$$
\phi_{x}=\frac{\mathscr{N}_{x}}{\sqrt{\left\langle B_{x}\right\rangle(x)\left\langle D_{x}\right\rangle(x)}} \exp \left(-\Omega_{T} \mathscr{S}(s) d s\right)
$$

207 where

$$
\mathscr{S}(s)=\int_{0}^{x} \log \frac{\left\langle D_{x}\right\rangle(s)}{\left\langle B_{x}\right\rangle(s)} d s .
$$

\section{${ }_{208}$ S.1.3 Deterministic model: Basins of attraction and bistability}

209 In order to understand the dependency of the deterministic model given by Eqs. (1)-(3) to parameters as well as the effect of 210 the initial amount of transcription factor (TF) in the achievement of the on or the off states, we have explored two 2-parametric 211 sections in the 3-parametric space $\left(B_{2}, B_{8}, K_{11}\right)$, both included in the bistability region, namely $K_{11}=94.155549$ and $B_{8}=30$. 212 For each section, we first determined the boundaries of the bistability region by using numerical continuation methods from ${ }_{213}$ XPP/AUTO (37), see Fig. A, violet solid curves. The bifurcation diagram displays the curve of saddle-node bifurcations: if we 
(a)

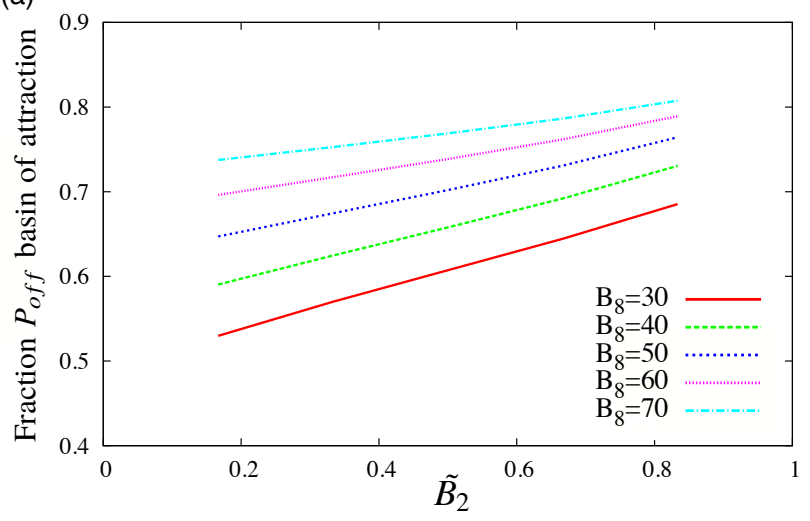

(b)

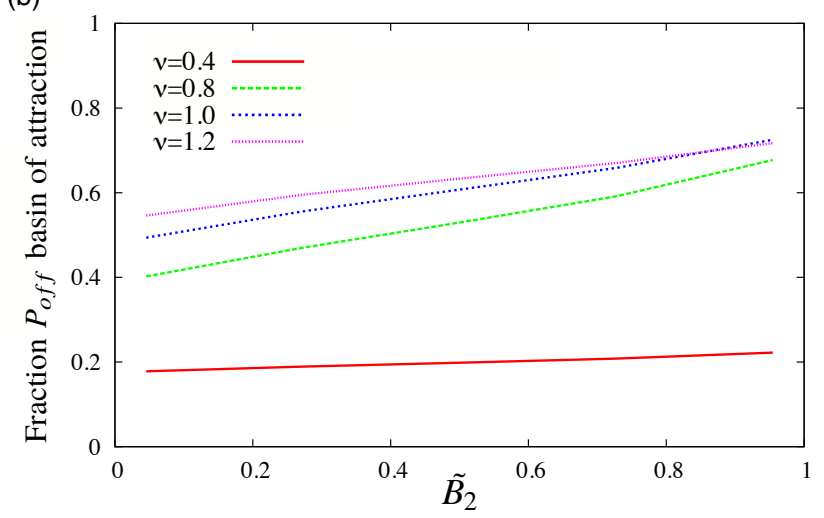

Figure B: Relative size of the basin of attraction of $P_{\text {off }}$ for parameter sets within the bistability region. Note that the size of the basin of attraction for $P_{o n}=1-P_{o f f}$. (a) One-dimensional representation in terms of $\widetilde{B}_{2}$ for 5 different values of $B_{8}$, with $K_{11}=94.155549$. (b) One-dimensional representation in terms of $\widetilde{B}_{2}$ for 4 different values of $K_{11}$, with $B_{8}=30$. As defined in (S.34), $\widetilde{B}_{2}$ stands for a normalisation of parameter $B_{2}$.

${ }_{214}$ let only one free parameter (say, $\left.B_{2}\right)$ and take the other parameters fixed ( $B_{8}$ and $K_{11}$ in this case), two saddle-node bifurcations 215 arise. Beyond both bifurcation values, the system is monostable; at the first bifurcation in the direction of increasing $B_{2}$, two 216 equilibria appear (one stable and another unstable) and the system becomes bistable (two stable equilibria) while, at the second 217 bifurcation, two equilibria disappear leading to a monostable system again. More specifically, the TF is at the ON state above 218 the bistability region in the space $\left(B_{2}, B_{8}\right)$, and reaches the OFF state below it (panel (a) in Fig. A). The situation is reversed in 219 the parameter space $\left(B_{2}, K_{11}\right)$ (see panel (b) in Fig. A).

220 To estimate the relative sizes of the basins of attraction in the bistability regimes we fix different values of $B_{8}$ and increase 221 parameter $B_{2}$ (see Fig. A, horizontal dotted lines). As mentioned, the two possible equilibrium states, $P_{\text {off }}$ and $P_{\text {on }}$, can be 222 achieved depending on the initial conditions of variables $x, q_{A}$, and $q_{I}$. For each specific choice of the parameter values, we 223 are interested in quantifying how likely a given initial number of TF molecules, of H3K4me3, and H3K27me 3 make the ${ }_{224}$ system to achieve the on or off state. To do so, we have simulated $24 \times 10^{6}$ initial conditions regularly sampled within the cube $225[0.005,3.00] \times[0.005,1.00] \times[0.005,1.00]$ in the $\left(x, q_{A}, q_{I}\right)$-space to determine the fraction of initial conditions that reach $P_{o f f}$ 226 (alternatively, $P_{o n}=1-P_{o f f}$ ). We take this fraction as an approximation of the size of the basin of attraction of each steady 227 state. For each initial condition we have numerically solved Eqs. (1-3) in the Main Manuscript with a fourth-order Runge-Kutta 228 method (with a constant time step-size $\delta t=10^{-2}$ ). The stabilisation of the solutions towards a given fixed point has been 229 established when the Euclidean norm of the vector field, $\|\mathscr{F}\|_{2}:=\left|\sqrt{\dot{x}^{2}+\dot{q A}^{2}+\dot{q}^{2}}\right|$, satisfies $\|\left.\mathscr{F}\right|_{2}<10^{-12}$.

${ }_{230}$ Figure B shows the relative size of the basin of attraction of $P_{\text {off }}$ for parameter sets within the bistability region. As in ${ }_{231}$ Fig. A, we study both the case $K_{11}=94.155549$ and the case $B_{8}=30$ (see panels (a) and (b), respectively). For the sake of ${ }_{232}$ comparison, for each $B_{8}$ or $K_{11}$ value, we have normalized the bistability interval to be of length 1 by using the information 233 about the two saddle-node bifurcations that bound the bistability region. We define a parameter

$$
\widetilde{B}_{2}:=\frac{B_{2}-B_{2, s n 1}}{\Delta B_{2}}
$$

234 where $B_{2, s n 1}$ (resp., $B_{2, s n 2}$ ) is the value of $B_{2}$ at the lowest saddle-node bifurcation and $\Delta B_{2}=B_{2, s n 2}-B_{2, s n 1}$ is the bistability inter${ }_{235}$ val length. In particular, for $K_{11}=94.155549$ and $B_{8}=30,40,50,60,70, B_{2, s n 1} \approx 16.0847,25.1832,36.2579,48.6356,61.6569$, ${ }_{236}$ respectively, and $\Delta B_{2} \approx 9.7056,12.5102,12.9733,11.5153,8.7999$, respectively. For $B_{8}=30$ and $K_{11}=94.155549 v$, for $v=$ ${ }_{237} 0.4,0.8,1.0,1.2$, we have that $B_{2, s n 1} \approx 83.1726,25.9152,16.0847,10.1504$, respectively, and $\Delta B_{2} \approx 7.4610,14.6055,9.7056,4.4709$, 238 respectively. In the main text we show interpolated surfaces of the values computed with this procedure (see Fig. 6 in the main 239 manuscript). 
Alarcón et al.
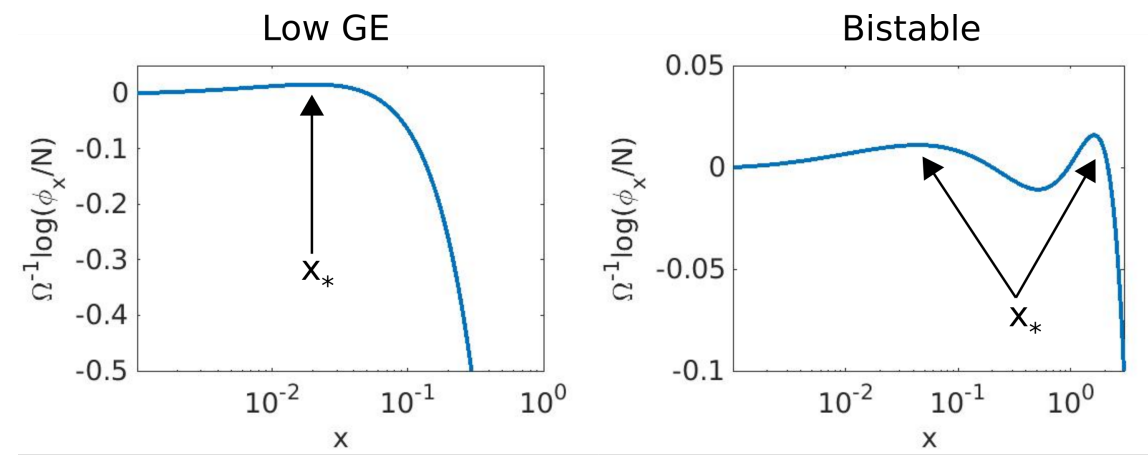

Noise-induced Bist.
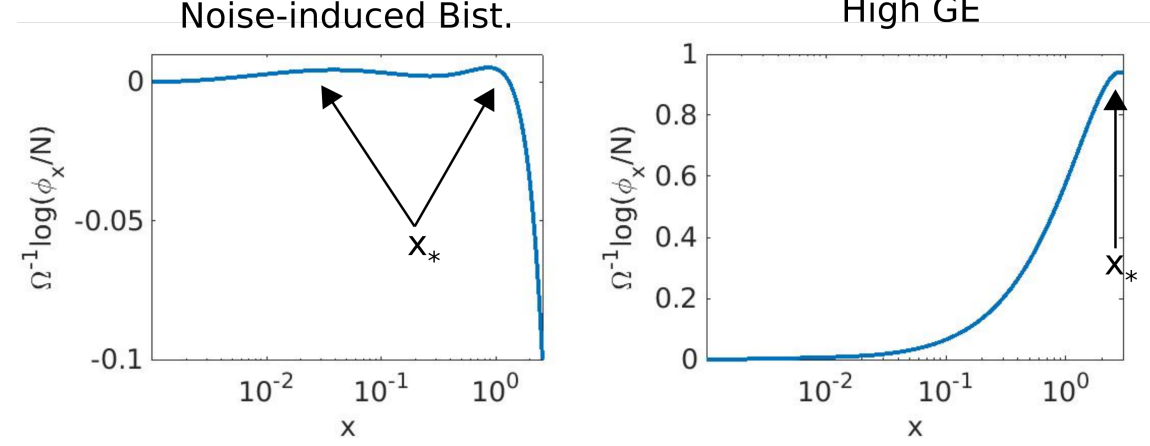

Figure C: $\phi_{x}(x)$ for different sets of parameter values representative of each sub-ensemble. For each case, we mark the corresponding value(s) of $x_{*}$.

\section{${ }_{240}$ S.1.4 Parameter sensitivity analysis: Ensemble approach}

\section{${ }_{241}$ S.1.4.1 Ensemble description}

242 The ensembles invoked in the Results section of the main text have been generated by a uniform sampling of a 16${ }_{243}$ dimensional parameter space. Each parameter set is thus described by a vector, $\theta$, in such space, whose components are:

$$
\theta=\left(A_{1}, A_{3}, B_{2}, K_{2}, K_{5}, K_{6}, \lambda_{A}, \lambda_{x}, A_{7}, A_{9}, B_{8}, K_{8}, K_{9}, K_{11}, K_{12}, \lambda_{I}\right)
$$

${ }_{244}$ (see Table B). Each component of $\theta$ is independently generated by sampling from a uniform prior distribution within a certain 245 prescribed range. The three different ensembles considered in this paper correspond to changes in the admissible range in ${ }_{246}$ (some of) the parameter values, as described in Table $\mathrm{C}$

Table C: Range of variation of each parameter within each of the ensembles.

\begin{tabular}{|c|c|c|c|c|c|c|c|}
\hline$A_{1}$ & $A_{3}$ & $B_{2}$ & $K_{2}$ & $K_{5}$ & $K_{6}$ & $\lambda_{A}$ & $\lambda_{x}$ \\
\hline$[0.01,10]$ & {$[0.001,0.1]$} & {$[0.001,100]$} & {$[0.01,400]$} & {$[0.01,400]$} & {$[0.1,10]$} & {$[0.1,10]$} & {$[0.1,10]$} \\
\hline$A_{7}$ & $A_{9}$ & $B_{8}$ & $K_{8}$ & $K_{9}$ & $K_{11}$ & $K_{12}$ & $\lambda_{I}$ \\
\hline$[0.01,10]$ & {$[0.001,1]$} & {$[0.001,100]$} & {$[0.01,200]$} & {$[0.01,200]$} & {$[0.01,400]$} & {$[0.1,10]$} & {$[0.1,10]$} \\
\hline
\end{tabular}

\section{${ }_{247}$ S.1.4.2 Classification into sub-ensembles}

${ }_{248}$ As described in Section S.1.4.1, each parameter set is generated at random by sampling each component (parameter) 249 independently from its corresponding prior distribution. Parameter values thus generated are then classified into three sub250 ensembles (see Fig. 2(a) of the main text):

1. Viability. A randomly generated parameter set, $\theta$, is first checked for viability regarding the uniqueness of the solution of the variational problem from Eq. (S.32). The solution of such problem corresponds to finding the positive real roots 
of a 4th degree polynomial. If the number of such roots is equal to one for all values of $x$, then $\theta$ is deemed as viable, otherwise it is rejected.

2. Monostable. Viable parameter sets such that both $\partial_{x} \mathscr{S}(x)=0$ and $\partial_{x} \mathscr{U}\left(x, \Omega_{T}\right)=0$ have one and only one solution are classified as monostable. Such parameter sets produce a monomodal steady state PDF of the gene expression level, $x$. Monostable parameter sets are themeselves classified into two subclasses:

- High Gene Expression. If $\theta$ is such that the mode of $\phi_{x}(x), x_{*}(\theta)$ (defined as the unique root of $\partial_{x} \mathscr{U}\left(x, \Omega_{T}\right)=0$, see Fig. C and Fig. 3 of the main text), satisfies $x_{*}(\theta) \geq x_{T H R}, \theta$ is classified as belonging to the High Gene Expression (High GE) sub-ensemble.

- Low Gene Expression. If $x_{*}(\theta)<x_{T H R}, \theta$ is classified as belonging to the Low Gene Expression (Low GE) sub-ensemble.

3. Bistable. Viable parameter sets such that both $\partial_{x} \mathscr{S}(x)=0$ and $\partial_{x} \mathscr{U}\left(x, \Omega_{T}\right)=0$ have three positive solutions are classified as bistable.

4. Noise-Induced Bistable. Viable parameter sets such that both $\partial_{x} \mathscr{S}(x)=0$ have one and only one positive solution and $\partial_{x} \mathscr{U}\left(x, \Omega_{T}\right)=0$ have three positive solutions are classified as noise-induced bistable (N.-I. Bistable).

Table D: Brief summary of the generated ensemble of $\theta$ s.

\begin{tabular}{|l|l|l|l|l|}
\hline Total Number (Viable) & High GE (\%) & Low GE (\%) & Bistable (\%) & Noise-Induced Bist. (\%) \\
\hline 19576 & 21.026 & 73.774 & 4.771 & 0.429 \\
\hline
\end{tabular}

In Table D we present a brief summary of the results obtained for our ensemble (Section S.1.4.1). Details of the ${ }_{268}$ (sub)ensemble generated are provided in Fig.2(a)-(c) and Fig. 6(a)-(b) in the main text and Fig. D, where we show the ${ }_{269}$ distributions (boxplots) for each parameter (i.e. each component of the vector $\theta$ ). We will use this data to detect which 270 parameters show statistically significant differences between sub-ensembles to determine which of them are key to observe the 271 behaviour associated with each sub-ensemble (high/low GE, bistability, noise-induced bistability) $(7,8)$.

${ }_{272}$ Analysis of statistically significant differences between sub-ensembles. We start our analysis by comparing the ${ }_{273}$ marginal posteriors PDFs corresponding to the different sub-ensembles (see Figs. D). The rationale for performing this 274 analysis is as follows (see $(7,8)$ for more details). The classification of parameter sets into sub-ensembles corresponds 275 to physical/biological different behaviours. We expect that by discerning which parameters present statistically significant 276 differences between sub-ensembles, we can identify which of them are key to produce the behaviour associated to a particular ${ }_{277}$ sub-ensemble $(7,8)$. For example, consider the marginal CDFs for the parameter $A_{3}$. By inspection, the marginal CDFs ${ }_{278}$ corresponding to the bistable sub-ensembles are clearly different from those corresponding to the monostable sub-ensembles 279 (high and low GE). Furthermore, the data shows a clear bias towards lower values of $A_{3}$ within the bistable sub-ensembles. ${ }_{280}$ These results suggest that $A_{3}$ is one of the key parameters involved in bistable behaviour and that, if we wish to drive a ${ }_{281}$ bistable-to-monostable transition changing $A_{3}$, a feasible strategy consists of increasing the value of $A_{3}(7,8)$. Here, we perform 282 this analysis in a systematic and quantitative way.

${ }_{283}$ In order to perform such comparison, we use the two-sample Kolmogorov-Smirnov (KS) test. Such test returns a decision ${ }_{284}$ regarding the null hypothesis that both data sets are drawn from the same continuous distribution. The result is $h=1$ if the test 285 rejects the null hypothesis at the prescribed significance level, and $h=0$ otherwise. The results are summarised in Table E.

${ }_{286}$ We first observe that the division of the monostable sub-ensemble into two sub-ensembles (high and low GE) is statistically ${ }_{287}$ meaningful. In Table E(a), where we present the summary of the comparison between the marginal CDFs for the high-GE and ${ }_{288}$ low-GE sub-ensembles, we see that the marginal distributions for all parameters exhibit statistically significant differences. ${ }_{289}$ Only for $K_{8}$ the statitiscal evidence for such difference is weaker, as shown by the corresponding $p$-values. In fact, for Ensemble 2902 the KS test cannot reject the null hypothesis.

291 We now proceed to compare the bistable sub-ensemble with the high GE and low GE sub-ensembles. The results are shown 292 in Table E(b)-(c). By simple visual inspection, we can tell that the marginal posteriors of the bistable sub-ensemble share more ${ }_{293}$ similarities with the High GE sub-ensemble than with the low GE sub-ensemble. Specifically, the KS test cannot reject the null 294 hypothesis for only two $\left(K_{9}\right.$ and $\left.\lambda_{I}\right)$ out of the sixteen parameters. Furthermore, for $\left(A_{1}, K_{5}\right.$, and $\left.K_{12}\right)$ the rejection of the null 

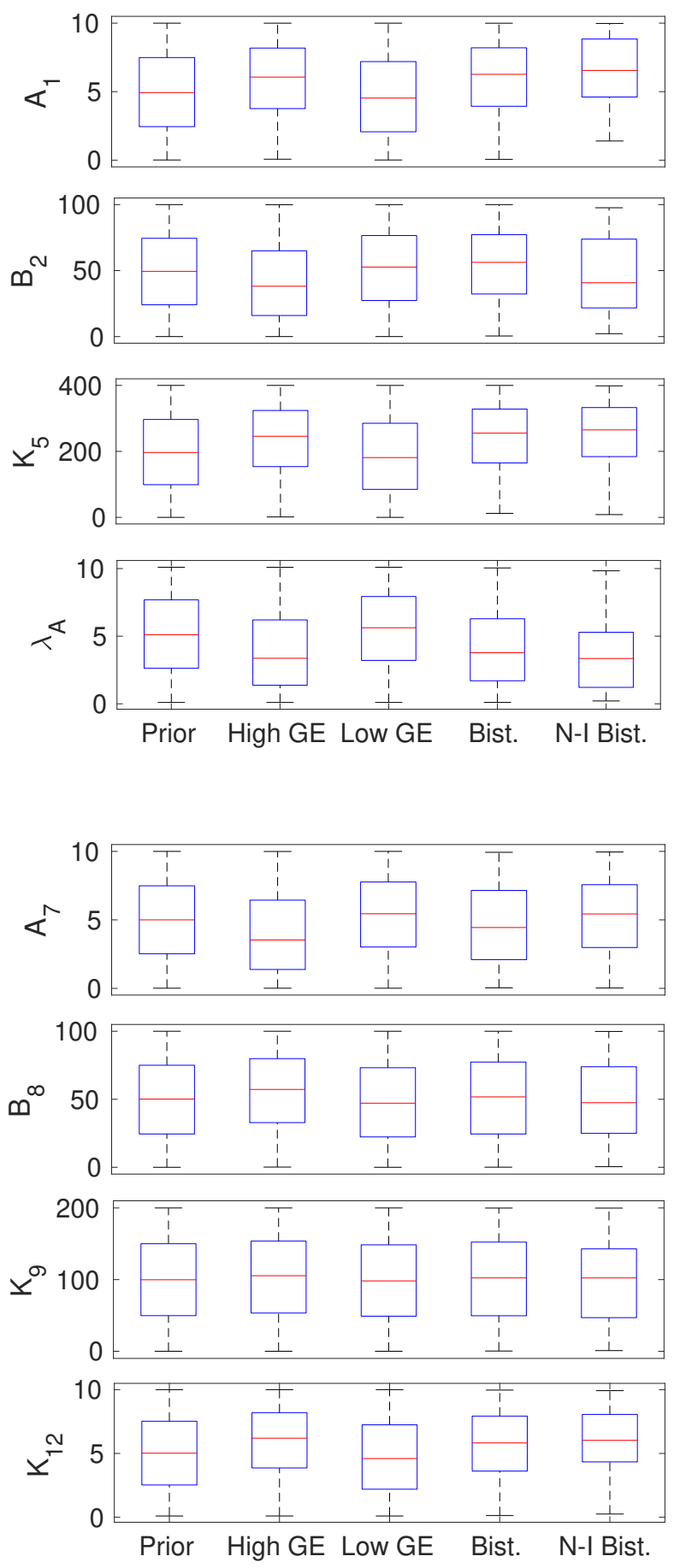
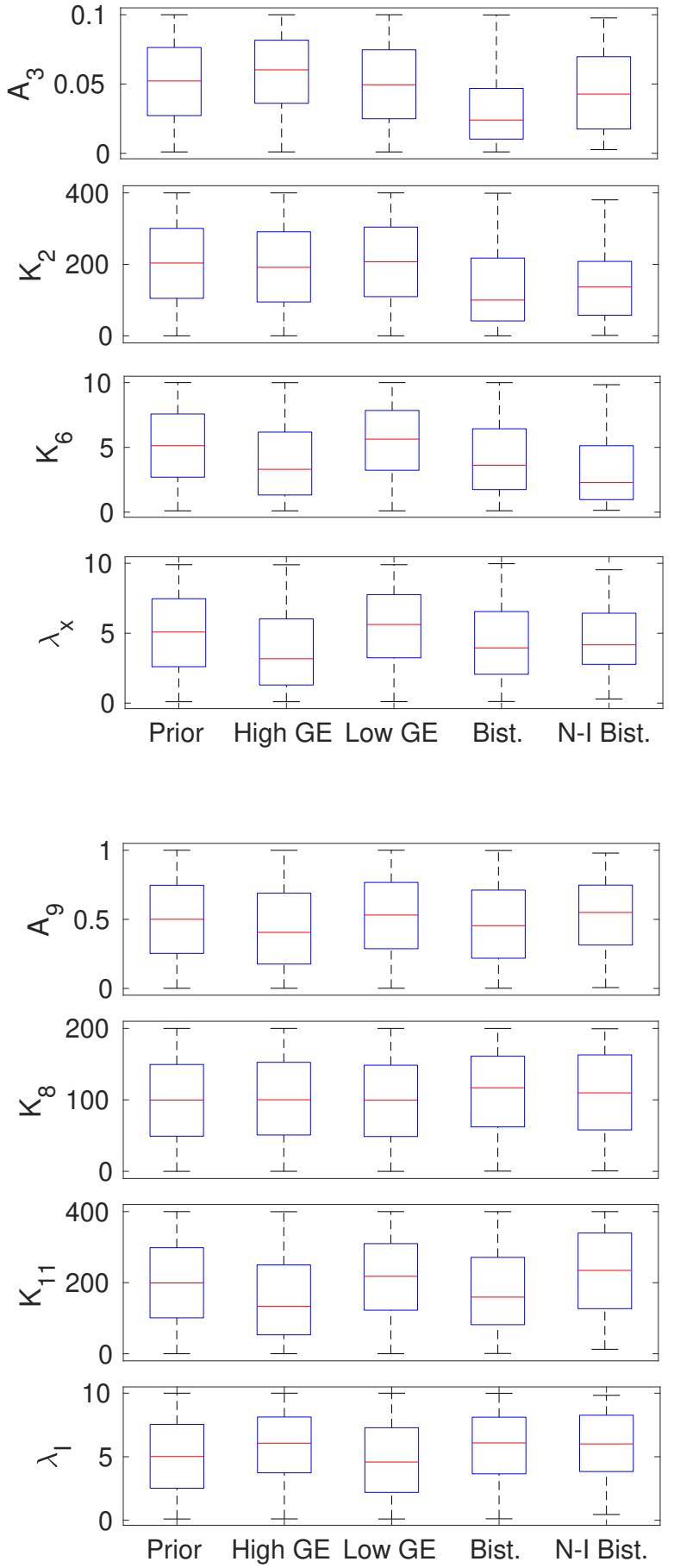

Figure D: Boxplots for the generated distribution of parameters. Each plot shows the boxplot corresponding to a specific parameter (see Table B) within each sub-ensemble. For reference, we also show the boxplot for the prior distribution. The upper (lower) panel shows results for parameters related to the modifiers of H3K4 (H3K27). 
Table E: Significance level $\alpha=0.075$.

(a) High vs Low GE

\begin{tabular}{|l|c|c|}
\hline & $h$ & p-value \\
\hline$A_{1}$ & 1 & $5.454601 \mathrm{e}-90$ \\
\hline$A_{3}$ & 1 & $5.870815 \mathrm{e}-46$ \\
\hline$B_{2}$ & 1 & $6.103946 \mathrm{e}-64$ \\
\hline$K_{2}$ & 1 & $3.503613 \mathrm{e}-06$ \\
\hline$K_{5}$ & 1 & $1.669834 \mathrm{e}-96$ \\
\hline$K_{6}$ & 1 & $1.056691 \mathrm{e}-166$ \\
\hline$\lambda_{A}$ & 1 & $1.282697 \mathrm{e}-155$ \\
\hline$\lambda_{x}$ & 1 & $1.885551 \mathrm{e}-188$ \\
\hline$A_{7}$ & 1 & $1.732416 \mathrm{e}-115$ \\
\hline$A_{9}$ & 1 & $2.317481 \mathrm{e}-53$ \\
\hline$B_{8}$ & 1 & $1.130947 \mathrm{e}-37$ \\
\hline$K_{8}$ & 1 & $5.041977 \mathrm{e}-02$ \\
\hline$K_{9}$ & 1 & $1.262770 \mathrm{e}-05$ \\
\hline$K_{11}$ & 1 & $3.614244 \mathrm{e}-143$ \\
\hline$K_{12}$ & 1 & $1.582725 \mathrm{e}-90$ \\
\hline$\lambda_{I}$ & 1 & $1.816639 \mathrm{e}-86$ \\
\hline
\end{tabular}

(b) High GE vs Bistable

\begin{tabular}{|l|c|c|}
\hline & $h$ & p-value \\
\hline$A_{1}$ & 1 & $7.471566 \mathrm{e}-02$ \\
\hline$A_{3}$ & 1 & $7.804065 \mathrm{e}-110$ \\
\hline$B_{2}$ & 1 & $4.189324 \mathrm{e}-27$ \\
\hline$K_{2}$ & 1 & $1.408325 \mathrm{e}-38$ \\
\hline$K_{5}$ & 1 & $6.347346 \mathrm{e}-02$ \\
\hline$K_{6}$ & 1 & $1.179497 \mathrm{e}-03$ \\
\hline$\lambda_{A}$ & 1 & $1.092038 \mathrm{e}-03$ \\
\hline$\lambda_{x}$ & 1 & $8.777037 \mathrm{e}-11$ \\
\hline$A_{7}$ & 1 & $9.895137 \mathrm{e}-08$ \\
\hline$A_{9}$ & 1 & $3.844402 \mathrm{e}-03$ \\
\hline$B_{8}$ & 1 & $2.351621 \mathrm{e}-05$ \\
\hline$K_{8}$ & 1 & $6.853621 \mathrm{e}-06$ \\
\hline$K_{9}$ & 0 & $1.786971 \mathrm{e}-01$ \\
\hline$K_{11}$ & 1 & $3.094784 \mathrm{e}-08$ \\
\hline$K_{12}$ & 1 & $1.587451 \mathrm{e}-02$ \\
\hline$\lambda_{I}$ & 0 & $6.137966 \mathrm{e}-01$ \\
\hline
\end{tabular}

(c) Low GE vs Bistable

\begin{tabular}{|l|l|c|}
\hline & $h$ & p-value \\
\hline$A_{1}$ & 1 & $1.962069 \mathrm{e}-31$ \\
\hline$A_{3}$ & 1 & $1.417666 \mathrm{e}-64$ \\
\hline$B_{2}$ & 1 & $8.579826 \mathrm{e}-04$ \\
\hline$K_{2}$ & 1 & $9.754648 \mathrm{e}-60$ \\
\hline$K_{5}$ & 1 & $2.332903 \mathrm{e}-37$ \\
\hline$K_{6}$ & 1 & $5.720654 \mathrm{e}-38$ \\
\hline$\lambda_{A}$ & 1 & $3.098753 \mathrm{e}-31$ \\
\hline$\lambda_{x}$ & 1 & $5.410590 \mathrm{e}-25$ \\
\hline$A_{7}$ & 1 & $4.027444 \mathrm{e}-10$ \\
\hline$A_{9}$ & 1 & $2.025408 \mathrm{e}-06$ \\
\hline$B_{8}$ & 1 & $1.832587 \mathrm{e}-02$ \\
\hline$K_{8}$ & 1 & $2.607908 \mathrm{e}-07$ \\
\hline$K_{9}$ & 0 & $5.258169 \mathrm{e}-01$ \\
\hline$K_{11}$ & 1 & $1.212437 \mathrm{e}-22$ \\
\hline$K_{12}$ & 1 & $1.952996 \mathrm{e}-20$ \\
\hline$\lambda_{I}$ & 1 & $1.002306 \mathrm{e}-21$ \\
\hline
\end{tabular}

(d) Bistable vs Noise-induced Bimodal

\begin{tabular}{|l|l|c|}
\hline & $h$ & p-value \\
\hline$A_{1}$ & 0 & $2.897352 \mathrm{e}-01$ \\
\hline$A_{3}$ & 1 & $3.662555 \mathrm{e}-04$ \\
\hline$B_{2}$ & 1 & $1.608322 \mathrm{e}-03$ \\
\hline$K_{2}$ & 0 & $1.115658 \mathrm{e}-01$ \\
\hline$K_{5}$ & 0 & $6.618277 \mathrm{e}-01$ \\
\hline$K_{6}$ & 1 & $8.472578 \mathrm{e}-03$ \\
\hline$\lambda_{A}$ & 0 & $2.219046 \mathrm{e}-01$ \\
\hline$\lambda_{x}$ & 0 & $9.567488 \mathrm{e}-02$ \\
\hline$A_{7}$ & 1 & $6.993023 \mathrm{e}-02$ \\
\hline$A_{9}$ & 0 & $1.994637 \mathrm{e}-01$ \\
\hline$B_{8}$ & 0 & $9.308129 \mathrm{e}-01$ \\
\hline$K_{8}$ & 0 & $9.117183 \mathrm{e}-01$ \\
\hline$K_{9}$ & 0 & $7.579813 \mathrm{e}-01$ \\
\hline$K_{11}$ & 1 & $4.657800 \mathrm{e}-03$ \\
\hline$K_{12}$ & 0 & $2.781216 \mathrm{e}-01$ \\
\hline$\lambda_{I}$ & 0 & $6.847780 \mathrm{e}-01$ \\
\hline
\end{tabular}

295 hypothesis is supported by weaker statistical evidence (see Table E(b)). For the low GE sub-ensemble, the number of such 296 parameters is reduced to three (data not shown).

297 Further analysis is carried out by comparing the bistable sub-ensembles (regular and noise-induced) as shown in Table E(d). ${ }_{298}$ Such an analysis produces the result that only 5 parameters exhibit statistically significant differences regarding their marginal 299 posteriors. Parameters $B_{2}$ and $K_{11}$ are specially relevant cases within such subset of parameters, as they are closely related to 300 ER enzyme activity and thus to the levels of positive and negative epigenetic marks, respectively.

\section{${ }_{301}$ S.1.5 Ensemble averages}

302 Recall that the steady state PDF $\phi_{x}(x)$, as given by Eq. (S.35):

$$
\phi_{x}(x \mid \theta)=\frac{\mathscr{N}_{x}}{\sqrt{\left\langle B_{x}\right\rangle(x)\left\langle D_{x}\right\rangle(x)}} \exp \left(-\Omega_{T} \mathscr{S}(x)\right)
$$

303 where

$$
\mathscr{S}(x)=\int_{0}^{x} \log \frac{\left\langle D_{x}\right\rangle(s)}{\left\langle B_{x}\right\rangle(s)} d s .
$$

${ }_{304}$ We have also made explicit in the notation that the steady-state PDF $\phi_{x}$ is conditioned to the choice of a particular set of 305 parameter values, $\theta$.

In order to calculate ensemble averages of positive and negative marks,

$$
\left\langle q_{A} \mid \theta\right\rangle_{x}=\int_{0}^{\infty} q_{A}(x) \phi_{x}(x \mid \theta) d x
$$

307 and

$$
\left\langle q_{I} \mid \theta\right\rangle_{x}=\int_{0}^{\infty} q_{I}(x) \phi_{x}(x \mid \theta) d x,
$$

308 respectively, we will need to approximate the value of the corresponding integrals, since in general we can give no closed exact ${ }_{309}$ expression. Since we are assuming that $\Omega_{T} \gg 1$, we apply the Laplace method to approximate such integrals. Such method 310 applies to integrals of the type:

$$
I\left(\Omega_{T}\right)=\int_{a}^{b} f(x) e^{-\Omega_{T} g(x)} d x .
$$


Alarcón et al.

${ }_{311}$ In the limit of $\Omega_{T} \gg 1$, if $g(x)$ exhibits a (unique) minimum at $x_{*} \in(a, b)$, the integral can be approximated by (35):

$$
I\left(\Omega_{T}\right) \approx \sqrt{\frac{2 \pi}{\Omega_{T} g^{\prime \prime}\left(x_{*}\right)}} f\left(x_{*}\right) e^{-\Omega_{T} g\left(x_{*}\right)} .
$$

${ }_{312}$ If $g(x)$ has two minima within $(a, b), x_{*_{-}} \in\left(a, x_{*_{0}}\right)$ and $x_{*_{+}} \in\left(x_{*_{0}}, b\right)$, where $x_{*_{0}}$ is the maximum of $g(x)$, we simply write:

$$
I\left(\Omega_{T}\right)=\int_{a}^{x_{*_{0}}} f(x) e^{-\Omega_{T} g(x)} d x+\int_{x_{*}}^{b} f(x) e^{-\Omega_{T} g(x)} d x
$$

${ }_{313}$ So that the integral can now be approximated by:

$$
I\left(\Omega_{T}\right) \approx \sqrt{\frac{2 \pi}{\Omega_{T} g^{\prime \prime}\left(x_{*_{-}}\right)}} f\left(x_{*_{-}}\right) e^{-\Omega_{T} g\left(x_{*_{-}}\right)}+\sqrt{\frac{2 \pi}{\Omega_{T} g^{\prime \prime}\left(x_{*_{+}}\right)}} f\left(x_{*_{+}}\right) e^{-\Omega_{T} g\left(x_{*_{+}}\right)} .
$$

${ }_{314}$ In our case, we have that $f(x) \equiv \frac{\mathscr{N}_{x}}{\sqrt{\left\langle B_{x}\right\rangle\left\langle D_{x}\right\rangle}} q_{J}(x)$, where $J=A, I, g(x)=\mathscr{S}(x)$, and

$$
g^{\prime \prime}=\mathscr{S}^{\prime \prime}=\frac{\left\langle D_{x}\right\rangle^{\prime}}{\left\langle D_{x}\right\rangle}-\frac{\left\langle B_{x}\right\rangle^{\prime}}{\left\langle B_{x}\right\rangle}
$$

315 where

$$
\left\langle B_{x}\right\rangle^{\prime} \equiv \frac{d\left\langle B_{x}\right\rangle}{d x}=\partial_{x}\left\langle B_{x}\right\rangle-\left.\frac{\partial_{x} G_{A}}{\partial_{p_{A}} G_{A}}\right|_{p_{A}=q_{A}^{*}} \partial_{p_{A}}\left\langle B_{x}\right\rangle-\left.\frac{\partial_{x} G_{I}}{\partial_{p_{I}} G_{I}}\right|_{p_{I}=q_{I}^{*}} \partial_{p_{I}}\left\langle B_{x}\right\rangle
$$

316 and

$$
\left\langle D_{x}\right\rangle^{\prime} \equiv \frac{d\left\langle D_{x}\right\rangle}{d x}=\partial_{x}\left\langle D_{x}\right\rangle-\left.\frac{\partial_{x} G_{A}}{\partial_{p_{A}} G_{A}}\right|_{p_{A}=q_{A}^{*}} \partial_{p_{A}}\left\langle D_{x}\right\rangle-\left.\frac{\partial_{x} G_{I}}{\partial_{p_{I}} G_{I}}\right|_{p_{I}=q_{I}^{*}} \partial_{p_{I}}\left\langle D_{x}\right\rangle .
$$

${ }_{317}$ Note that, since we cannot give expressions for $q_{A}^{*}(x)$ and $q_{I}^{*}(x)$ in closed analytical form, we have used the implicit function ${ }_{318}$ theorem and the fact that $q_{J}^{*}(x)$ satisfies $G_{J}\left(x, q_{J}^{*}\right)=B_{J}\left(x, q_{J}^{*}\right)-D_{J}\left(x, q_{J}^{*}\right)=0(J=A, I)$.

${ }_{319}$ Using these results, we can compute the ensemble-averaged positive and negative marks for both the monostable sub${ }_{320}$ ensembles (Low GE and High GE) and the bistable sub-ensemble

$$
\begin{aligned}
\left\langle q_{A}\right\rangle_{H} & =\frac{1}{N_{H}} \sum_{\theta \in H G S E}\left\langle q_{A} \mid \theta\right\rangle_{x},\left\langle q_{I}\right\rangle_{H}=\frac{1}{N_{H}} \sum_{\theta \in H G S E}\left\langle q_{I} \mid \theta\right\rangle_{x}, \\
\left\langle q_{A}\right\rangle_{L} & =\frac{1}{N_{L}} \sum_{\theta \in L G S E}\left\langle q_{A} \mid \theta\right\rangle_{x},\left\langle q_{I}\right\rangle_{L}=\frac{1}{N_{L}} \sum_{\theta \in L G S E}\left\langle q_{I} \mid \theta\right\rangle_{x}, \\
\left\langle q_{A}\right\rangle_{B} & =\frac{1}{N_{B}} \sum_{\theta \in B S E}\left\langle q_{A} \mid \theta\right\rangle_{x},\left\langle q_{I}\right\rangle_{B}=\frac{1}{N_{B}} \sum_{\theta \in B S E}\left\langle q_{I} \mid \theta\right\rangle_{x},
\end{aligned}
$$

${ }_{321}$ where the subindexes $H, L$, and $B$ refer to average with respect to the High GE (HGSE), Low GE (LGSE), and bistable 322 (BSE) sub-ensembles, respectively. $N_{H}, N_{L}$, and $N_{B}$ are the cardinals of the High GE, Low GE, and bistable sub-ensembles, ${ }_{323}$ respectively.

\section{${ }_{324}$ S.2 SUPPLEMENTARY TABLES AND FIGURES}

\section{REFERENCE}

1. Keener, J., and J. Sneyd, 1998. Mathematical physiology. Springer-Verlag, New York, NY, USA.

2. Ingalls, B. P., 2013. Mathematical modelling in systems biology. An introduction. The MIT Press, Cambridge, Massachusets, USA.

3. Dodd, I. B., M. A. Micheelsen, K. Sneppen, and G. Thon, 2007. Theoretical analysis of epigenetic cell memory by nucleosome modification. Cell 129:813-822.

4. Sneppen, K., M. A. Michelson, and I. B. Dodd, 2008. Ultrasensitive gene regulation by positive feedback loops in nucleosome modification. Mol Syst Biol. 4:182. 
Table F: Parameter set 1 (Monostable Low GE with $A_{3} \min$ ). $\rho_{A}=1, \rho_{I}=1 . \varepsilon_{1}=0.1$

\begin{tabular}{|c|}
\hline Methylation/demethylation of H3K4 \\
\hline$A_{1}=4.795239, A_{3}=0.001006, B_{2}=64.091515, K_{2}=171.650858, K_{5}=169.260923$ \\
\hline$K_{6}=4.256390, \lambda_{A}=8.658754$ \\
\hline Methylation/demethylation of H3K27 \\
\hline$A_{7}=7.110925, A_{9}=0.726804, B_{8}=89.408867, K_{8}=95.702027, K_{9}=28.886043$ \\
\hline$K_{11}=279.510584, K_{12}=8.484769, \lambda_{I}=0.808614$ \\
\hline Gene regulatory network \\
\hline$C_{1}=150, C_{2}=50, C_{4}=1000, \lambda_{X}=7.504438$ \\
\hline
\end{tabular}

Table G: Parameter set 2 (Bistability). $\rho_{A}=1, \rho_{I}=1 . \varepsilon_{1}=0.1$

\begin{tabular}{|c|}
\hline Methylation/demethylation of H3K4 \\
\hline$A_{1}=8.666099, A_{3}=0.040570 B_{2}=20.016911, K_{2}=271.229406, K_{5}=229.948007$ \\
\hline$K_{6}=6.207151, \lambda_{A}=7.194034$ \\
\hline Methylation/demethylation of H3K27 \\
\hline$A_{7}=3.800899, A_{9}=0.063084, B_{8}=30.087626, K_{8}=88.410503, K_{9}=59.322552$ \\
\hline$K_{11}=94.155549, K_{12}=6.854684, \lambda_{I}=1.310467$ \\
\hline Gene regulatory network \\
\hline$C_{1}=150, C_{2}=50, C_{4}=1000, \lambda_{X}=1.455620$ \\
\hline
\end{tabular}

5. Menéndez, J. A., B. Corominas-Faja, E. Cuyàs, M. G. García, S. Fernández-Arroyo, A. F. Fernández, J. Joven, M. F. Fraga, and T. Alarcón, 2016. Oncometabolic nuclear reprogramming of cancer stemness. Stem Cell Reports 6:273-283.

6. Loubiere, V., A.-M. Martinez, and G. Cavalli, 2019. Cell fate and developmental regulation dynamics by polycomb proteins and 3D genome architecture. BioEssays 41:1800222.

7. Folguera-Blasco, N., E. Cuyàs, J. A. Menéndez, and T. Alarcón, 2018. Epigenetic regulation of cell fate reprogramming in aging and disease: A predictive computational model. PLoS Comp. Biol. 14:e1006052.

8. Folguera-Blasco, N., R. Pérez-Carrasco, E. Cuyás, J. A. Menéndez, and T. Alarcón, 2019. A multiscale model of epigenetic heterogeneity reveals the kinetic routes of pathological cell fate reprogramming. PLoS Comp Biol. 15:e1006592.

9. Thalheim, T., M. Herberg, M. Loeffler, and J. Galle, 2017. The Regulatory Capacity of Bivalent Genes- A Theoretical Approach. Int J Mol Sci. 18(5):1069.

10. Thalheim, T., L. Hopp, H. Binder, G. Aust, and J. Galle, 2018. On the Cooperation between Epigenetics and Transcription Factor Networks in the Specification of Tissue Stem Cells. Epigenomes 2:20.

11. Anderson, D. F., and T. G. Kurtz, 2010. Stochastic analysis of biochemical systems. Springer, New York, NY, USA.

12. Haseltine, E. L., and J. B. Rawlings, 2002. Approximate simulation of coupled fast and slow reactions for stochastic chemical kinetics. J Chem Phys. 117:6959-6969.

13. Rao, C. V., and A. P. Arkin, 2003. Stochastic chemical kinetics and the quasi-steady-state assumption: Application to the Gillespie algorithm. J Chem Phys. 118:4999-5010.

14. Cao, Y., D. T. Gillespie, and L. R. Petzold, 2005. Multiscale stochastic simulation algorithm with stochastic partial equilibrium assumption for chemically reacting systems. J Comp Phys. 206:395-411.

15. Cao, Y., D. T. Gillespie, and L. R. Petzold, 2005. The slow-scale stochastic simulation algorithm. J Chem Phys. 122:014116.

16. Goutsias, J., 2005. Quasiequilibrium approximation of fast reaction kinetics in stochastic biochemical systems. J Chem Phys. 122:184102. 
Alarcón et al.

Table H: Parameter set 3 (Noise-induced bistability). $\rho_{A}=1, \rho_{I}=1 . \varepsilon_{1}=0.1$

\begin{tabular}{|c|}
\hline Methylation/demethylation of H3K4 \\
\hline$A_{1}=6.563150, A_{3}=0.040022, B_{2}=19.965753, K_{2}=248.998412, K_{5}=290.668266$ \\
\hline$K_{6}=2.846470, \lambda_{A}=1.419607$ \\
\hline Methylation/demethylation of H3K27 \\
\hline$A_{7}=4.389568, A_{9}=0.346774, B_{8}=63.121059, K_{8}=110.921072, K_{9}=20.259211$ \\
\hline$K_{11}=330.633279, K_{12}=9.438870, \lambda_{I}=2.983005$ \\
\hline Gene regulatory network \\
\hline$C_{1}=150, C_{2}=50, C_{4}=1000, \lambda_{X}=5.607232$ \\
\hline
\end{tabular}

Table I: Parameter set 4 (Monostable. High GE. $A_{3}$ min.). $\rho_{A}=1, \rho_{I}=1 . \varepsilon_{1}=0.1$

\begin{tabular}{|c|}
\hline Methylation/demethylation of H3K4 \\
\hline$A_{1}=3.720782, A_{3}=0.001024, B_{2}=7.810833, K_{2}=244.894942, K_{5}=169.260923$ \\
\hline$K_{6}=0.158431, \lambda_{A}=5.960934$ \\
\hline Methylation/demethylation of H3K27 \\
\hline$A_{7}=6.595129, A_{9}=0.530692, B_{8}=66.009204, K_{8}=14.539354 K_{9}=155.153281$ \\
\hline$K_{11}=122.173786, K_{12}=9.148418, \lambda_{I}=5.431558$ \\
\hline Gene regulatory network \\
\hline$C_{1}=150, C_{2}=50, C_{4}=1000, \lambda_{X}=1.701816$ \\
\hline
\end{tabular}

355 17. Ball, K., T. G. Kurtz, L. Popovic, and G. Rempala, 2006. Asymptotic analysis of multi-scale approximations to reaction networks. Ann App Prob. 16:1925-1961.

357 18. E, W., D. Liu, and E. Vanden-Eijnden, 2007. Nested stochastic simulation algorithms for chemical kinetic systems with multiple time scales. J Comp Phys. 221:158-170.

359 19. Mastny, E. A., E. L. Haseltine, and J. B. Rawlings, 2007. Two classes of quasi-steady state model reductions for stochastic kinetics. J Chem Phys. 127:094106.

361 20. Kang, H.-W., and T. G. Kurtz, 2013. Separation of time-scales and model reduction for stochastic reaction networks. Ann App Prob. 23:529-583.

363 21. Alarcón, T., 2014. Stochastic quasi-steady state approximations for asymptotic solutions of the Chemical Master Equation. J Chem Phys. 140:184109.

365 22. Kang, H.-W., T. G. Kurtz, and L. Popovic, 2014. Central limit theorems and diffusion approximation for multiscale Markov $366 \quad$ chain models. Ann App Prob. 24:721-759.

367 23. de la Cruz, R., P. Guerrero, F. Spill, and T. Alarcón, 2015. The effects of intrinsic noise on the behaviour of bistable $368 \quad$ systems in quasi-steady state conditions. J Chem Phys. 143:074105.

369 24. Ibánez-Marcelo, E., and T. Alarcón, 2016. Surviving evolutionary escape on complex genotype-phenotype networks. $J$ $370 \quad$ Math Biol. 72:623-647.

371 25. Ethier, S.N., and T. Kurtz, 1986. Markov processes: Characterization and Convergence. John Wiley \& Sons, Inc.

372 26. de la Cruz, R., P. Guerrero, J. Calvo, and T. Alarcón, 2017. Coarse-graining and hybrid methods for efficient simulation of 373 stochastic multi-scale models of tumour growth. J Comp Phys. 350:974-991.

374 27. Gardiner, C. W., 2009. Stochatic methods. Springer-Verlag, Berlin, Germany.

375 28. Horsthemke, W., and R. Lefever, 2006. Noise-induced transitions. Springer-Verlag, New York, NY, USA.

376 29. Bruna, M., S. J. Chapman, and M. J. Smith, 2014. Model reduction for slow-fast stochastic systems with metastable 377 behaviour. J Chem Phys. 140:174107. 

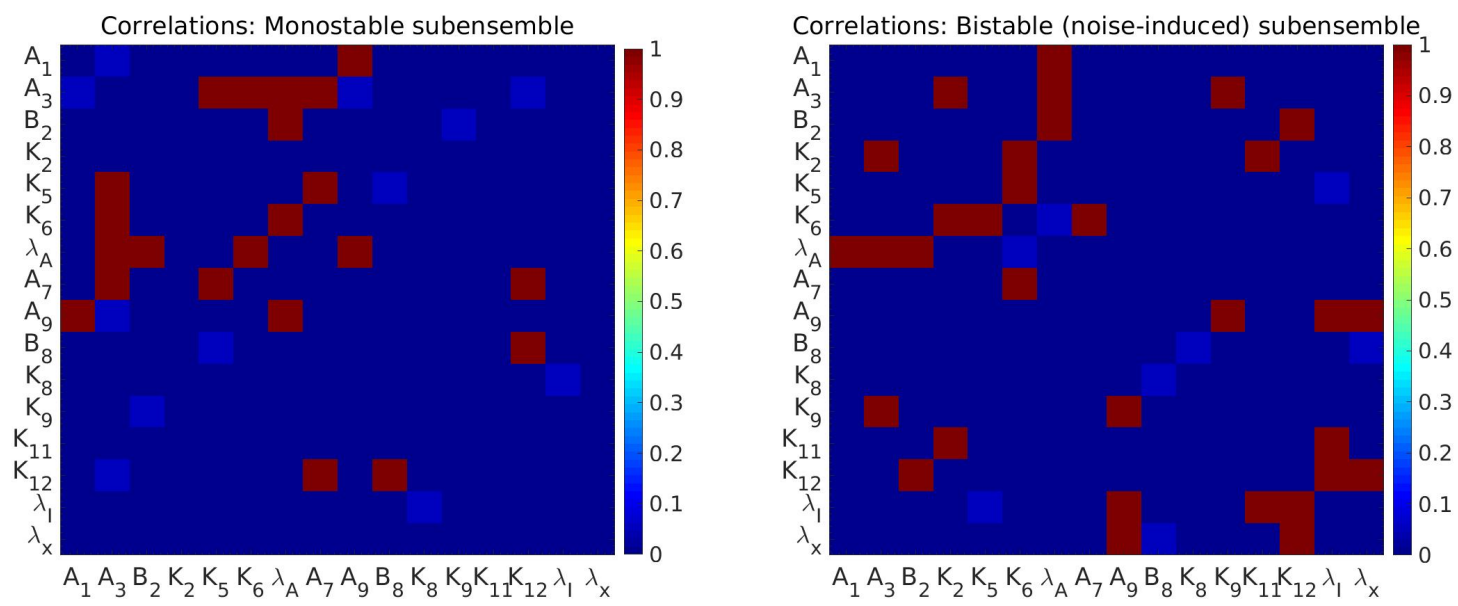

Figure E: Correlations within the different sub-ensembles. Red squares correspond to pairs such that the null hypothesis that the parameters are uncorrelated can be rejected ( $\mathrm{p}$-value $<0.05$ ), dark blue, corresponds to p-values $>0.5$ (uncorrelated pairs), and light blue, to $\mathrm{p}$-values $\gtrsim 0.05$, i.e. pairs of parameters for which the evidence of absence of correlations is weaker.

378 30. Hinch, R., and S. J. Chapman, 2005. Exponentially slow transitions on a Markov chain: the frequency of calcium sparks. Eur J Appl Math. 16:427-446.

380 31. Bressloff, P. C., 2014. Stochastic processes in cell biology. Springer-Verlag, Berlin, Germany.

381 32. Stakgold, I., 1998. Green's functions and boundary value problems. 2nd Ed. Wiley-Interscience, New York, NY, USA.

382 33. Ward, M. J., 1999. Exponential asymptotics and convection-reaction-diffusion models. In J. Cronin, and R. E. O’Mailley, editors, Analysing multiscale phenomena using singular perturbation methods. American Mathematical Society, Providence, RI, USA, 162-195.

385 34. Kogan, O., M. Khasin, B. Meerson, D. Schneider, and C. R. Myers, 2014. Two-strain competition in quasineutral stochastic disease dynamics. Phys Rev E 90:042149.

387 35. Murray, J. D., 1984. Asymptotic analysis. Springer-Verlag, New York, NY, USA.

38в 36. Biancalani, T., and M. Assaf, 2015. Genetic toggle switch in the absence of cooperative binding: Exact results. Phys Rev Lett. 115:208101.

390 37. Ermentrout, B., 2002. Simulating, Analyzing, and Animating Dynamical Systems. Society for Industrial and Applied 391 Mathematics. http://www.math.pitt.edu/ bard/xpp/xpp.html.

392 38. Sneeringer CJ, Scott MP, Kuntz KW, Knutson SK, Pollock RM, Richon VM, et al. Coordinated activities of wild-type plus mutant EZH2 drive tumour-associated hypertrimethylation of lysine 27 on histone H3 (H3K27) in human B-cell lymphomas. Proc Natl Acad Sci U.S.A. 2010; 107(49): 20980-20985. 
Alarcón et al.
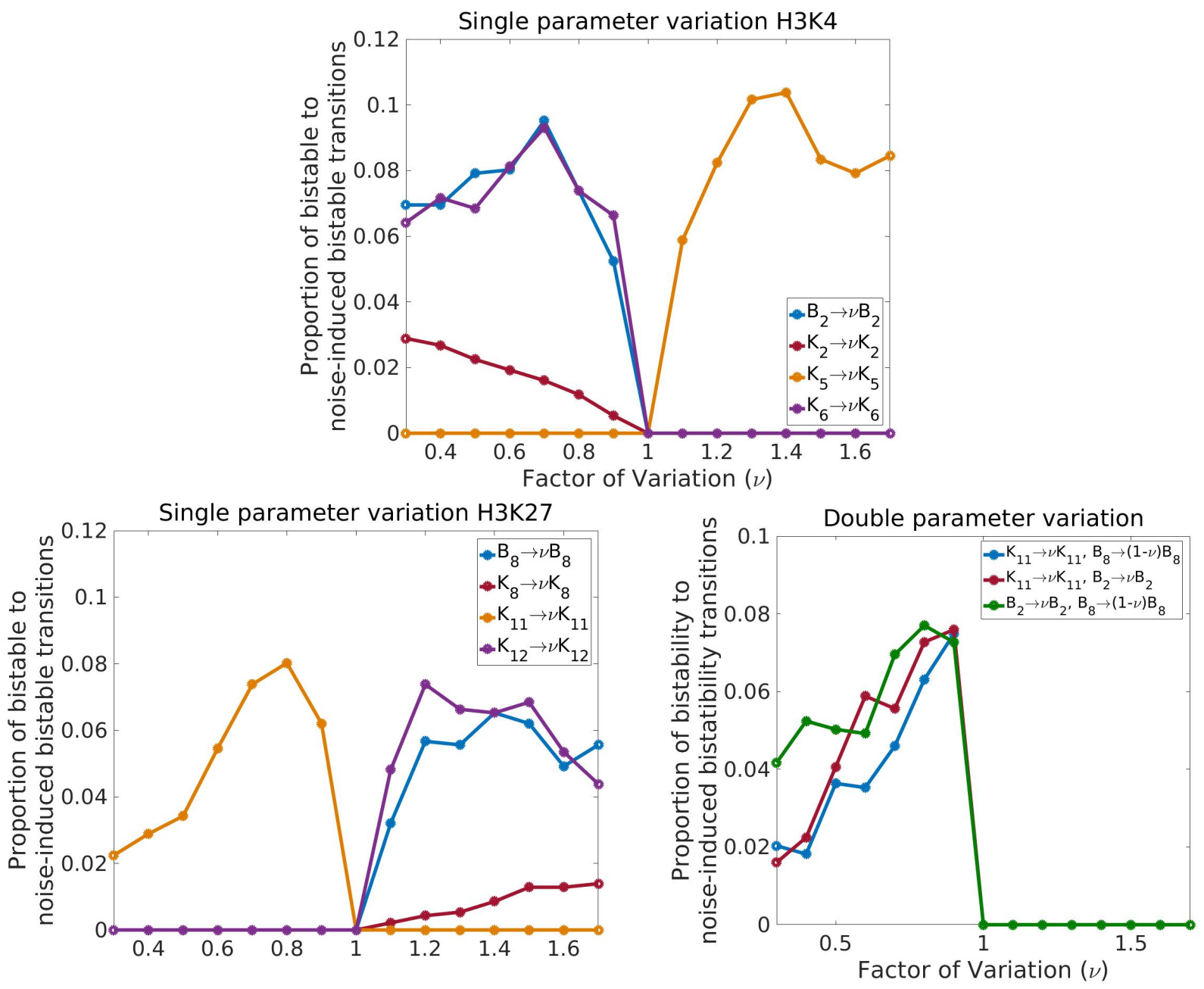

Figure F: Further sensitivity analysis for the Bistable sub-ensemble. These plots show our results regarding the sensitivity of the systems within the Bistable sub-ensemble to variations of selected parameters. Here we measure the probability that a system within the Bistable sub-ensemble moves to the Noise-Induced Bistable sub-ensmeble as a function of the factor of variation, $v$. 

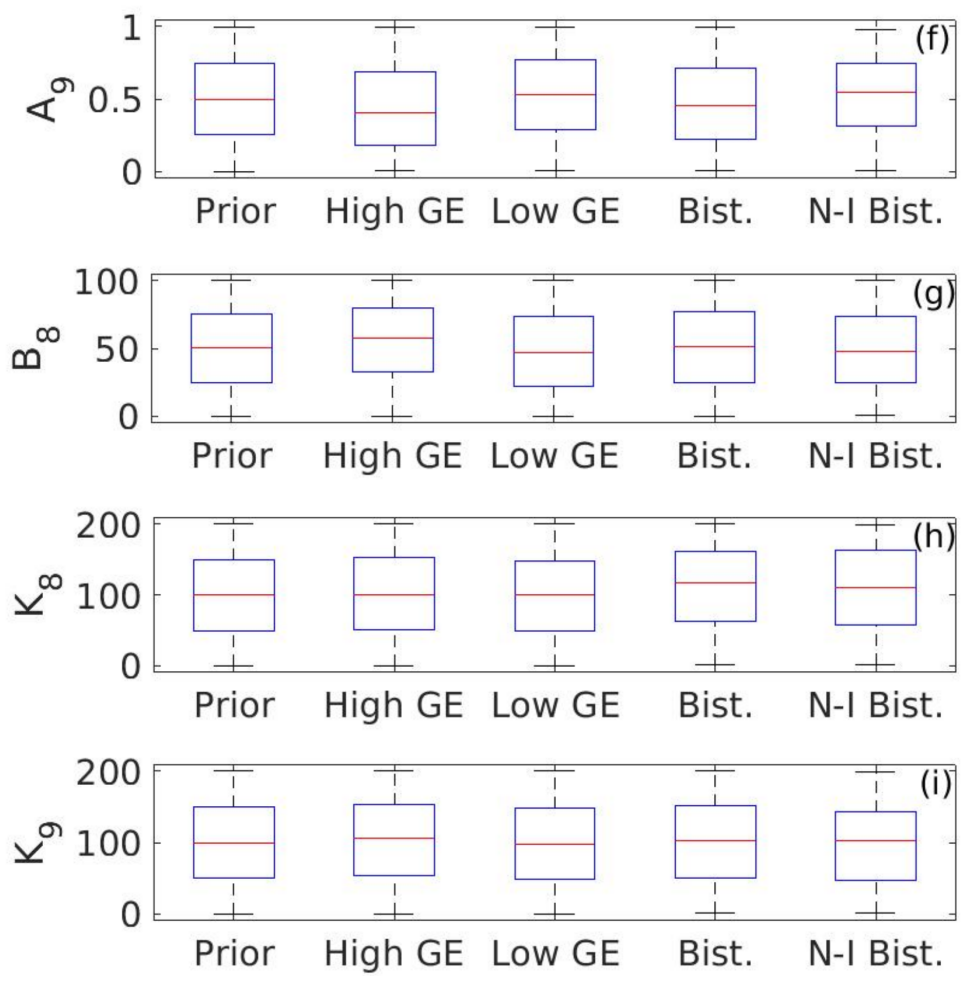

Figure G: Statistics of specific parameters related to EZH2 enzymatic activity. Quantitative analysis performed for four parameters related to the enzymatic kinetics of EZH2 (specifically $K_{M}$-like parameters, see Eq. (3) of the main text) for each of the four sub-ensembles shows no obvious differences between the Low GE PDFs and those corresponding to the High GE and the Bistable sub-ensembles. Statistical analysis shows significant differences, but with $p$-values very close to the significance level. This observation is consistent with direct in vitro measurements of the Michaelis-Menten constant, $K_{M}$, and the catalytic constant, $k_{c a t}$, of the wild-type EZH2 and Y641F and Y641N mutants by Ref. (38), who found that in all variants of the enzyme, the $K_{M} \mathrm{~s}$ retained similar values within the error bars. 